\title{
Dynamics of Planetary Systems in Star Clusters
}

\author{
R. Spurzem ${ }^{1}$, M. Giersz 2 , D.C. Heggie $^{3}$, D. N. C. Lin ${ }^{4,5}$ \\ 1) Astronomisches Rechen-Institut, Zentrum für Astronomie, University of Heidelberg, \\ Mönchhofstr. 12-14, 69120 Heidelberg, Germany \\ 2) Nicolaus Copernicus Astronomical Centre, Bartycka 18, 00-716 Warszawa, Poland \\ 3) University of Edinburgh, School of Mathematics and Maxwell Institute for Mathematical \\ Sciences, King's Buildings, Mayfield Rd., Edinburgh EH9 3JZ, Scotland, UK \\ 4) UCO/Lick Observatory, University of California, Santa Cruz, CA 95064, USA \\ 5) Kavli Institute for Astronomy and Astrophysics, Peking University, Beijing, China \\ spurzem@ari.uni-heidelberg.de
}

\begin{abstract}
At least $10-15 \%$ of nearby sun-like stars have known Jupiter-mass planets. In contrast, very few planets are found in mature open and globular clusters such as the Hyades and 47 Tuc. We explore here the possibility that this dichotomy is due to the post-formation disruption of planetary systems associated with the stellar encounters in long-lived clusters. One supporting piece of evidence for this scenario is the discovery of freely floating low-mass objects in star forming regions. We use two independent numerical approaches, a hybrid Monte Carlo and a direct $N$-body method, to simulate the impact of the encounters. We show that the results of numerical simulations are in reasonable agreement with analytical determinations in the adiabatic and impulsive limits. They indicate that distant stellar encounters generally do not significantly modify the compact and nearly circular orbits. However, moderately close stellar encounters, which are likely to occur in dense clusters, can excite planets' orbital eccentricity and induce dynamical instability in systems which are closely packed with multiple planets. The disruption of planetary systems occurs primarily through occasional nearly parabolic, non adiabatic encounters, though eccentricity of the planets evolves through repeated hyperbolic adiabatic encounters which accumulate small-amplitude changes. The detached planets are generally retained by the potential of their host clusters as free floaters in young stellar clusters such as $\sigma$ Orionis. We compute effective cross sections for the dissolution of planetary systems and show that, for all initial eccentricities, dissolution occurs on time scales which are longer than the dispersion of small stellar associations, but shorter than the age of typical open and globular clusters. Although it is much
\end{abstract}


more difficult to disrupt short-period planets, close encounters can excite modest eccentricity among them, such that subsequent tidal dissipation leads to orbital decay, tidal inflation, and even disruption of the close-in planets.

Subject headings: globular cluster, extra-solar planets - solar system: evolution

\section{Introduction}

The most successful observational method for planet searches has been the method of radial velocity surveys. Among over 1000 target stars, Jupiter- mass planets, with period up to a few years, have been found around more than $10 \%$ of them (Cumming et al. 2007). This fraction is likely to increase with follow-up high-precision measurements of trends in the radial velocity curves which reveal suggestive signals for many additional planetary companions.

This prolific success in the discovery of planets around these field stars is in sharp contrast to the rarity of planets around stars in both open and globular clusters. Despite several attempts to search for planets in the Hyades (Guenther et al. 2005), only one planet is found around one star (Sato et al. 2007). It is tempting to attribute this dichotomy to an environmental influence on the proficiency of planet formation. For example, Bonnell et al. (2001) suggested that, if the cluster went through an initial high density phase, close stellar encounters during the planetary formation epoch may have tidally truncated the disk and eliminated the domain of giant planet formation. This argument, however, is weakened by the poorly known time scale and location of giant planet formation. It also does not take into account the possibility of subsequent stellar accretion of the tidally stripped gas which may provide a protracted environment for planet formation. The discovery of planets in several binary systems with separation of a few AU's (Queloz et al. 2000, Hatzes et al. 2003, Zucker et al. 2004, Correia et al. 2007) is another sign that perturbations which are induced by neighboring stars alone cannot be an efficient mechanism to quench planet formation.

The current paradigm for star formation theory is based on the realization that field stars are generally formed in dense gaseous environments which are similar to, but slightly less massive and concentrated than the progenitor of the longer-lived open clusters. In many dense star-forming molecular clouds, the fraction of stars with protostellar disks depends more sensitively on the stellar age rather than the dynamical properties of their host clusters (Hillenbrand 2005). Even in dense regions which contain massive stars, such as the Orion nebula, the silhouettes of protostellar disks are commonly found (O'Dell et al. 1993). Traces of radioactive decay products in the most primitive chondritic meteorites provide strong evidence that the formation of the solar system was preceeded by a nearby supernova event 
(Cameron \& Truran 1977). Since the massive progenitors of supernovae are only found in relatively dense molecular clouds, it is likely that the solar system actually formed in a stellar aggregate with 2000 members (Adams \& Laughlin 2001). These circumstantial pieces of evidence support the conjecture that planets may not form less prolifically in open clusters than the progenitors of the present-day field stars.

An alternative scenario for the rarity of planet detection in open clusters may be attributed to post-formation dynamics. There is supporting circumstantial evidence for dynamical disruption of planetary systems during the infancy of their host stars in dense molecular clouds. A population of "freely floating" planets, with 5-15 Jupiter masses, has been found in the young cluster $\sigma$ Orionis (Zapatero-Osorio et al. 2000, Lucas et al. 2001). If these free floaters indeed formed near some host stars, a reliable census of their frequency in a wide range of star forming regions would provide a calibration on the critical conditions for planetary system disruption. However, this task is particularly challenging because their detection and distinction from bound planets by microlensing observations are generally difficult (c.f. Han 2006).

In contrast to open clusters, stellar associations which provided the birth place for the known planet-bearing field stars probably dispersed under the combined action of internal dynamical relaxation, stellar evolution, and the tidal perturbation of their host galaxies over a time scale $\sim 10^{8}$ yr. During this epoch, the planetary-retention probability of individual stars is determined by a competition between cluster disruption and planetary-system break up. The planetary-system dichotomy would be explained if stellar interactions and encounters affect the stability and dynamical evolution of their companion planetary systems on a time scale longer than the associations' dispersal time scale but shorter than the age of typical open clusters.

On the theoretical side, Smith \& Bonnell (2001) inferred that only a small fraction of planets would become detached from their host stars during the characteristic life span of young clusters and association. Their results are obtained using the equations of restricted three body motion to approximate encounters between planetary systems with nearly circular orbits and single stars. They claimed that the few detached planets would escape from open and young clusters because the planets' recoil speed is generally large compared with the clusters' velocity dispersion. These conclusions are modified if the perturbers are binary stars. This problem has been systematically addressed with a Monte Carlo approach by Laughlin \& Adams (1998) in which they focused their attention on the effect of four body scattering (a binary star, and a planet). They averaged the stellar density over the cluster to obtain effective cross sections for the disruption of a Jupiter-like planetary orbit by all the binary stars. Also Davies \& Sigurdsson (2001) included in their Monte Carlo study 
encounters with binaries.

In these early studies, a restricted range of planetary orbits was under consideration. For example, Laughlin and Adams focused their attention on planets which were initially on a 5 AU circular orbit around the host star. However, the known extra solar planets have a semimajor axis ranging from $0.05 \mathrm{AU}$ to several AU. Those with periods longer than a week have a nearly uniform eccentricity distribution up to unity (cf. Cumming et al., in prep.). Although planets are probably formed in their nascent disks beyond the snow line with nearly circular orbits (Ida \& Lin 2004), their eccentricity may be excited shortly after their formation through their interaction with their nascent disks (Goldreich \& Sari 2003, Creswell et al. 2007) or with each other (Rasio \& Ford 1996, Lin \& Ida 1997). Dynamical instability and relaxation is particularly effective in exciting the planets' eccentricity especially in closelypacked multiple-planet systems (Papaloizou \& Terquem 2002, Zhou et al 2007, Juric \& Tremaine 2007, Ford \& Rasio 2007).

For most non-disruptive encounters, the stellar perturbation nonetheless modifies the eccentricities of the planets. Individual planets with modestly eccentric orbits may be more vulnerable to disruption by subsequent stellar perturbations. Repeated stellar interactions may be particularly damaging to the survival of planetary systems. Observational data also indicate that a significant fraction of known planets, if not most of them, have additional planetary siblings around their host stars (Fischer et al. 2001). Although the present-day separation in these known systems is generally wide and they are intrinsically stable, stellar perturbation in the past can provide an additional avenue for inducing their dynamical relaxation. The cumulative excitation of eccentricity, which results from both close and distant single and binary star encounters, may also lead to dynamical instabilities in closely packed multiple planetary systems, resulting in dissolution of a planetary system or in the merger of some planets with their host star. The destabilization of these multiple-planet systems generally occurs through secular and nonlinear interactions over many orbital periods (Zhou et al. 2007).

Taking these physical processes into account, there is a need to investigate the effect of stellar encounters on planetary systems with more general initial condition than previously considered. Preliminary studies (see e.g. the different results obtained from direct $N$-body simulations of Hurley \& Shara 2002) indicate that the planetary retention efficiency may be strongly modified if the planets attain a modest eccentricity shortly after their birth. Ideally, the dynamical interaction between multiple planets and cluster stars needs to be investigated. In reality, the vast range of possible orbital configuration and mass distribution make any attempts to represent potential planetary systems futile. The orbital periods of compact planetary systems are much shorter than the crossing time of the cluster. On the time 
scale of a large number of orbital periods, multiple planets interact with each other through cumulative low-amplitude secular perturbations. The accuracy requirement for calculating the long-term behaviour of multiple-planet systems is much higher than that needed for the reliable simulation of star clusters. For long-term solar system dynamics, symplectic methods, using a generalized leap-frog, like the widely used Wisdom-Holman symplectic mapping method (Wisdom \& Holman 1991, see also review by Duncan \& Quinn 1993), are the best suited integration methods. They do not show secular errors in energy and angular momentum. In principle, the symplectic mapping methods can be used to treat the planets, while the dynamics and perturbation induced by their host stars is computed with an $N$ body scheme. However, in their standard implementation, the symplectic mapping methods require a constant time step, which is not compatible with the central motivation of the Ahmad-Cohen neighbour scheme. Another more practical approach to strongly reducing the planets' secular errors in a $N$-body scheme is to enforce a time-symmetric scheme by making the time steps reversible through an iteration (Hut, Makino, \& McMillan 1995, Funato et al. 1996, Kokubo, Yoshinaga \& Makino 1998). Such schemes have not yet been used for long-term secular evolution of planetary systems.

Because of these complications, it would be immensely challenging to consider the effects of secular interactions between closely-spaced multiple planets concurrently with the dynamical evolution of the cluster. As an alternative, we may aim to disentangle the different physical effects, and we begin here by focusing solely on the influence of gravitational encounters on an otherwise unperturbed planetary orbit. Having derived cross sections and time scales for these processes as functions of relevant parameters of the planetary system and its environment, we will be in a much better position to assess the conditions under which internal and external perturbations of planetary systems will couple, and those in which they will act on very different time scales.

This analysis can also be applied to address the issue of a lack of planets in globular clusters. In a recent search for transit events among stars in the core region of an old, metalpoor globular cluster, 47 Tuc, no short-period planets have been found (Weldrake 2007 and references therein), even though 17 such objects were expected to be discovered (Gilliland et al. 2000). One possible cause for the absence of planets in globular clusters such as 47 Tuc may be the suppression of their formation due to lack of heavy elements in their progenitor clouds (Ida \& Lin 2005a). However, we note that the mean metallicity deficiency (compared with the solar value) of 47 Tuc is smaller than the spread in the dust mass among disks around T Tauri stars (Beckwith 1998). The detection of another planet around a pulsar in globular cluster M4 confirms that although their formation may be suppressed it is not prohibited around metal-deficient stars. However, its stellar allegiance may have been altered through an exchange/ capture event during a close encounter between its original parent and 
its present host star (Sigurdsson et al. 2003).

Short-period (a few days) planets reside deeply in the potential of their host stars and can only be perturbed by a very close encounter between stars. Using an order of magnitude estimate for the encounter time scale between a single star and a planetary system, Bonnell et al. (2001) argued that, in globular clusters, planets with semi major axis greater than 0.3 AU may be detached from their host stars. This estimate is based on an extrapolation from the outcomes of encounters between systems of three bodies of comparable mass, which may not be appropriate for the limiting case in which one of these bodies (the planet) is much less massive than the others (Fregeau et al. 2006).

In dense globular clusters, occasional close stellar encounters can induce eccentricity excitation even for planets with relatively short periods (Davies \& Sigurdsson 2001). In multiple-planet systems, eccentricity excitation of long-period planets can also affect closein planets through the secular interaction between them (cf Mardling \& Lin 2002, Nagasawa \& Lin 2005). In addition, tidal dissipation inside both short-period planets and their host stars can damp their eccentricity as indicated by the negligible eccentricity observed among all planets with period $<6-7$ days (cf Dobbs-Dixon et al. 2004). The combined influence of these effects heats the planetary interior and may cause the planet to inflate (Bodenheimer et al. 2001). Very close to their host stars, tidal inflation may be sufficiently strong to disrupt planets ( $\mathrm{Gu}$ et al. 2003). As the stellar relaxation process leads to an increase in the density of background stars, their perturbation on their planetary companions intensifies. This process may be particularly effective in eliminating planetary companions of relatively low-mass stars in a globular cluster environment such as 47 Tuc.

In order to 1) verify this induced disruption scenario for the differences between planetary systems around stars inside and outside stellar clusters, 2) quantitatively determine the critical condition for planetary retention, and 3) assess the impact of stellar perturbations on close-in planets, we consider, in this paper, the dynamical evolution of planet-bearing stars in a range of cluster environments. In contrast to previous investigations, we focus on the diverse dynamical structure of planetary systems rather than the distribution of stellar kinematics, mass, and multiplicity. For simplicity of analysis, we only consider interactions between single stars and planetary systems. In realistic clusters where their fraction is large, binary stars may indeed provide more effective perturbers than single stars for the break-up of planetary systems, especially during the early epoch of cluster evolution when the stellar density is relatively high. Nevertheless, interactions between single stars are also important for the long-term survival of planetary systems in star clusters.

In order to resolve some of these outstanding issues in both open and globular cluster environments, we carry out a series of numerical simulations which are designed to investi- 
gate the influence of stellar encounters on the dynamics of extrasolar planets with a wide variety of orbital properties. In contrast to previous studies we use a special $\mathrm{N}$-body code (NBODY6++: Spurzem 1999), which is based on the NBODY6 code by Aarseth (1999a,b, 2003) and can be used on massively parallel computers. In addition we use the hybrid Monte Carlo model (HMC) of Giersz \& Spurzem (2003) as an approximate model of star clusters with large particle numbers and many planetary systems.

These simulations, though more time consuming than plain statistical approaches in which only encounters are modeled, have the advantage that they can take into account both the spatial and time variation of the stellar background in young, open, and globular clusters. In a previous series of experiments, Davies \& Sigurdsson (2001) have studied a Monte Carlo model of planetary systems interacting with stars and binaries in a dense star cluster such as 47 Tuc. However they did not include the dynamical evolution of the cluster and just studied isolated three- and four-body encounters. (Also their coverage of parameters was smaller than ours.) Both the NBODY6++ and HMC code are well suited to follow a wide range of encounters and to overcome some of these shortcomings.

The NBODY6++ scheme is also ideally suited to the inclusion of the dominant dynamical influence of binary stars and occasional hierarchical triple systems, but in this paper we only focus on interactions of single stars with planetary systems. Another advantage is the possibility of simulating the consequence of several successive stellar encounters. After the planetary eccentricities are slightly excited by the first encounters, their rate of increase may be accelerated during the subsequent encounters.

In this paper, we first focus on a comparison between empirical cross sections for orbital changes and analytical estimates (using several approximations). This study is intended to clarify the extent to which the existing analytical cross sections can be used, and we also are interested to see from the numerical models where they cannot be used any more, e.g. for the case of liberation of planets, either by successions of weak encounters or single strong ones. Our main interest is to understand the physical mechanisms, and an improvement for more realistic environments (binaries, multi-planetary systems and their internal interactions) is the subject of future work. Section 2 summarizes some of the existing knowledge on analytical cross sections for changes of eccentricity and semi-major axis of a planetary system due to encounters. In Section 3 we describe the setup of the numerical simulations and Section 4 contains a description and analysis of the results. We pay special attention to the changes of planetary orbits in a statistical way by searching for encounter events which affect their eccentricities and semi-major axes (see for comparison also Theuns 1996). Once identified, data on these events are accumulated, and the results are then binned and presented as empirical differential cross sections for the outcomes of encounters between single stars and 
planetary systems; here we use methods developed for the Monte-Carlo models and those of Giersz \& Spurzem (2003). Finally, we summarize the findings of these numerical experiments and discuss their astrophysical implications in Section 5.

\section{Analytical Results on Encounters with Planetary Systems}

\subsection{General Analytical Approach}

We aim to study the statistics of encounters between a passing star (the third body) and a simple planetary system consisting of a single planet and its star. We focus on the change in eccentricity $\delta e$ and the relative change in binding energy $\Delta=\delta \varepsilon / \varepsilon$. These depend on all the initial parameters of the encounter, but the essential parameters are the distance of closest approach, $r_{p}$, and the speed of the third body at infinity, $V$ (see Figs. 1 and 2). If $r_{p} \gg a$, where $a$ is the semi-major axis of the planet, then the encounter is tidal. If $V$ is much larger than the orbital speed of the planet then the encounter is impulsive, unless $r_{p}$ is so large that the time scale of the encounter becomes comparable with the period of the planet. At still larger $r_{p}$ the encounter is adiabatic, which means here that the angular speed of the planet is much larger than that of the passing star. If $V$ is small compared to the orbital speed of the planet, then a tidal encounter is always adiabatic. When $r_{p}$ is very large the path of the passing star is hyperbolic with high eccentricity, but at smaller values (though still in the tidal regime $r_{p} \gg a$ ) it is nearly parabolic. These distinctions are essential in the analytical interpretation of numerical data.

Appendix A collects a number of formulae for $\Delta$ and $\delta e$ which are approximately valid deep within some of these regimes. In adiabatic regimes an important role is played by parameters $e^{\prime}$ and $K$, which serve to quantify these ideas. The first parameter is just the eccentricity of the relative orbit of the passing star. The parameter $K$ is defined by

$$
K=\sqrt{\frac{2 M_{12}}{M_{123}}\left(\frac{r_{p}}{a}\right)^{3}}
$$

where $r_{p}$ denotes the distance of pericentre for the passing star, the mass of the binary is $M_{12}=m_{1}+m_{2}$, the mass of the third star is $m_{3}$, and $M_{123}=M_{12}+m_{3}$. In our simulations, $m_{1}=m_{3}$ and $m_{2} \ll m_{1}$, and so we have $K=\left(r_{p} / a\right)^{3 / 2}$. $K$ measures the ratio of the time scales involved, and a necessary condition for an adiabatic encounter in the sense explained above is $K \gg 1$. However, this criterion by itself would be sufficient only if the encounter with the third star were parabolic. If the encounter is hyperbolic the condition for an adiabatic encounter is given by $K / \sqrt{e^{\prime}+1} \gg 1$ (Heggie \& Rasio 1996), in which the 
hyperbolic eccentricity

$$
e^{\prime}=\sqrt{1+\left(\frac{p V^{2}}{G M_{123}}\right)^{2}},
$$

where $p, V$ denote the impact parameter and relative velocity at infinity. With these parameters we get for the minimum distance $r_{p}$ of the third star relative to the centre of mass of the planetary system

$$
r_{p}=\frac{G M_{123}}{V^{2}}\left(e^{\prime}-1\right) .
$$

The impact parameter $p$ and distance of closest approach $r_{p}$ are related by

$$
p=r_{p} \sqrt{1+\frac{2 G M_{123}}{r_{p} V^{2}}} .
$$

Now we consider the orbital response of a planet as a consequence of stellar encounters. Due to perturbations by passing stars, the semi-major axis and eccentricity of a planet experiences successive changes $\delta a$ and $\delta e$ which correspond to changes in its binding energy and angular momentum per unit mass, according to the equations

$$
\begin{gathered}
\Delta=\frac{\delta E}{E}=-\frac{\delta a}{a} \\
\frac{\delta J}{J}=\frac{1}{2} \frac{\delta a}{a}-\frac{e \delta e}{1-e^{2}} .
\end{gathered}
$$

The orbital energy per unit mass $E$ is inversely proportional to the semi-major axis, and for a circular orbit it is just minus half the squared velocity. Accompanying the changes in $e$ and $a$ are changes in $i, \omega$, and $\Omega$, but we pay no attention to these in this paper.

The formulae in Appendix A give approximate expressions for $\Delta$ and $\delta e$ for a single encounter, and depend on all the initial conditions. For statistical purposes it is more useful to consider instead cross sections, $\sigma$. For example $\sigma(\Delta)$, for $\Delta>0$, would be defined as the cross section for encounters in which the relative change of binding energy exceeds $\Delta$. This would be a function of $V, a$, the masses and (possibly) $e$, though usually we will consider cross sections averaged over the distribution of $e$. Often also we consider differential cross sections such as $d \sigma / d \Delta$.

Cross sections essentially involve the impact parameter $p$, while the size of the changes $\Delta$ and $\delta e$ is more directly related to the distance of closest approach, $r_{p}$. There are two limiting cases: (i) strongly hyperbolic encounters without significant gravitational focusing, where $V^{2} \gg G M_{123} / p$, so that $e^{\prime} \gg 1, r_{p} \gg G M_{123} / V^{2}$, and hence $p \propto r_{p}$; and (ii) the case in which $V^{2} \ll G M_{123} / p, e^{\prime} \approx 1$, and so $r_{p} \ll G M_{123} / V^{2}$. In this second case we have 
strong gravitational focusing and the second term in equation (4) above dominates, so that $p \propto \sqrt{r_{p}}$. When $V=0$ the encounter is parabolic and $e^{\prime}=1$.

A given planetary system may experience encounters of either type, but which type dominates depends essentially on $V$ and $a$. We have performed simulations for two ranges of semi-major axes of planetary orbits, which we denote for reference as "hard" (0.03 to 5 AU) and "soft" (3-50 AU), cf. Table 1. As is shown in Figs. 1 and 2 our "soft" planets nearly exclusively experience encounters where the velocity $V$ of the encountering star at infinity is larger than the orbital velocity of the planet $v_{\text {orb }}$. In the case of "hard" planets (in our sense) we find a large amount of encounters (but not all) with the opposite case $V<v_{\text {orb }}$. Note that our use of the term "hard" and "soft" for planetary systems differs from the definition

of Fregeau et al. (2006). They use the velocity $v_{c}$ as a threshold, such that for $V \approx v_{c}$ the binding energy of the planetary system is comparable to the kinetic energy of the incoming star. Such definition is equivalent to what is used for binaries in star clusters (Heggie 1975), where hard binaries have a clear tendency to become harder in close encounters. However, in our models, the planetary mass is very small compared to the stellar mass $\left(m_{2} \ll m_{1}\right)$, so we have $v_{c} \approx v_{\text {orb }} m_{2} / m_{1} \ll v_{\text {orb }}$. An inspection of our Figs. 1 and 2 shows that there are practically no encounters with $V<v_{c}$. Therefore we choose our definition of "hard" and "soft" planets, which we will use henceforth without quotation marks, just motivated to distinguish two different initial sets of semi-major axes of planetary systems. Indeed, in our models there are effectively only two regimes. For our soft planets, $V$ typically exceeds the orbital speed of the planet $V>v_{\text {orb }}$. These encounters are impulsive if $r_{p}$ is not too large. They become adiabatic and hyperbolic if $r_{p}$ is large enough. On the other hand, many (but not all) encounters of our hard planets fall into the category $V<v_{\text {orb }}$. Here tidal encounters at small enough $r_{p}$ are parabolic, but hyperbolic encounters occur at large enough $r_{p}$.

\subsection{Cross Sections for the Change of Eccentricity}

\subsubsection{Impulsive encounters}

In the case of soft planets, the most important tidal encounters may be treated as impulsive. First we provide an estimate for the form of the cross section, and then state an accurate result.

Let $\tau$ be the time scale for the encounter, i.e. $\tau=r_{p} / V_{p}$, where $V_{p}$ is the velocity of the passing star at pericentre. Our assumption here is that $\tau \lesssim t_{\text {orb }}$, where $t_{\text {orb }}$ is the orbital time of the planetary system, i.e. we are far from the fully adiabatic limit. Then we approximate 
the change of velocity of the orbiting planet due to the perturbation by the passing star as

$$
\delta v \approx \tau \delta a=\frac{4 G m_{3} a}{V_{p} r_{p}^{2}}
$$

where $\delta a$ is the difference between the acceleration of the planet (by the perturber) and that of the parent star (by the perturber), which we estimate by a tidal approximation.

Now the relative changes in the binding energy, $\varepsilon$, and angular momentum, $J$, of the planetary system may be estimated by $\delta v / v$, and so by equation (6) this may also be used to estimate $D$, which we define as the change in the square of the eccentricity, $D=\delta\left(e^{2}\right)$. (This is more convenient than $\delta e$ itself in some later analysis.) So we have

$$
D \sim \frac{\delta v}{v} \approx \sqrt{\frac{G m_{3}}{a V_{p}^{2}}} \cdot\left(\frac{a}{r_{p}}\right)^{2}
$$

For the last step above we have used $m_{3} \approx m_{1}$. We also use $V_{p}^{2}=V^{2}+2 G\left(m_{1}+m_{3}\right) / r_{p}$. Thus

$$
D \approx \sqrt{\frac{G m_{3}}{a V_{p}^{2}}} \cdot\left(\frac{a}{r_{p}}\right)^{2} \propto r_{p}^{-2} .
$$

The total cross section is $\sigma=\pi p^{2} \simeq \pi r_{p}^{2} \propto D^{-1}$, and so we get for the differential cross

$$
\frac{d \sigma}{d D} \mid \propto D^{-2}
$$

For a proper estimate we may adopt the approach which was taken by Heggie (1975) for the computation of the cross section for the relative energy change. As shown in Appendix B.1, the result is that

$$
\frac{d \sigma}{d D}=2 C_{1} \frac{\left(G m a^{3}\right)^{1 / 2}}{V D^{2}}
$$

where we have specialized to the case in which the two stars (the passing star and the sun in the planetary system) have the same mass $m$, and $C_{1}$ is a constant which is defined in terms of a certain average, and evaluates numerically to $C_{1}=0.883$ approximately.

\subsubsection{Adiabatic encounters}

Now we consider adiabatic tidal encounters. Using a first-order expansion, Heggie (1975) and Heggie \& Rasio (1996) obtained the net secular (i.e. long-term relative to the binary's orbital period) changes of eccentricity $\delta e$ for parabolic and hyperbolic encounters between a 
single field star and a close binary star. We adapt their formula to our case, in which the masses $m_{1}$ of the planet's host star and $m_{3}$ of the approaching star are large compared to the planetary mass $m_{2}$; so we have $M_{12} \equiv m_{1}+m_{2} \approx m_{1}$ and $M_{123} \approx m_{1}+m_{3}$. equation (7) of Heggie \& Rasio (1996) yields for this case

$$
\delta e=-\frac{15}{4}\left(\frac{m_{3}^{2}}{m_{1} M_{123}}\right)^{1 / 2}\left(\frac{a}{r_{p}}\right)^{3 / 2} \frac{e \sqrt{1-e^{2}}}{\left(1+e^{\prime}\right)^{3 / 2}} \quad g\left(e^{\prime}, \Omega, \omega, i\right),
$$

where $g$ is the function in curly brackets in eq. (A3) in the present paper. It reduces to $g=$ $\pi \sin (2 \Omega) \sin ^{2} i$ for parabolic encounters, so to order of magnitude we use $g \approx 1$ in this case. For highly hyperbolic encounters we have similarly $g \approx e^{\prime}, f_{3}\left(e^{\prime}\right)=e^{\prime} /\left(1+e^{\prime}\right)^{3 / 2} \approx e^{\prime-0.5}$, and we use $e^{\prime} \propto p \approx r_{p} \propto K^{2 / 3}$. It follows that

$$
\begin{aligned}
& \delta e_{\text {par }} \propto K^{-1} \propto r_{p}^{-3 / 2} \\
& \delta e_{\text {hyp }} \propto K^{-4 / 3} \propto r_{p}^{-2}
\end{aligned}
$$

From equations (13) we can deduce approximate total cross sections for both limits, using $\sigma \propto p^{2} \propto r_{p}^{2}$ for the hyperbolic case, and $\sigma \propto p^{2} \propto r_{p}$ for the parabolic case. It follows that

$$
\begin{aligned}
\sigma_{\mathrm{par}} & \propto(\delta e)^{-2 / 3} \\
\sigma_{\text {hyp }} & \propto(\delta e)^{-1}
\end{aligned}
$$

and so we recover in the parabolic case the form of the result in Heggie \& Rasio (1996) (see below). From this, differential cross sections are computed by

$$
\begin{aligned}
& \left.\frac{d \sigma}{d(\delta e)}\right|_{\text {par }} \propto(\delta e)^{-5 / 3} \\
& \left.\frac{d \sigma}{d(\delta e)}\right|_{\text {hyp }} \propto(\delta e)^{-2}
\end{aligned}
$$

The details are given in Appendix B, where the following accurate results are obtained. (Note, however, that these are differential cross sections for the change in $e^{2}$, i.e. $D=\delta\left(e^{2}\right)$.) In the case of extremely hyperbolic encounters with hard planets, the calculation is carried out in Appendix B.2, and yields a formula of the same form as for impulsive encounters:

$$
\frac{d \sigma}{d D}=\frac{16 C_{2}}{3} \frac{\sqrt{G m a^{3}}}{V D^{2}}
$$

where $C_{2}=0.5932$ approximately. For parabolic encounters, however, the corresponding result was essentially given by equation (19) of Heggie \& Rasio (1996). As shown in Appendix B.3 this leads to the differential cross section

$$
\frac{d \sigma}{d D}=\frac{2}{21}(15 \pi)^{2 / 3}\left[\Gamma\left(\frac{2}{3}\right) \Gamma\left(\frac{5}{6}\right)\right]^{2} \frac{G m a}{V^{2}}(D)^{-5 / 3} .
$$

The form of this cross section is different, because of gravitational focusing. 


\subsection{Cross sections for the change in energy}

\subsubsection{Impulsive encounters}

We consider now the relative change in energy, $\Delta$, and begin with impulsive tidal encounters. As already mentioned, formulae were given by Section 4.2 of Heggie (1975) for the change in energy in an impulsive tidal encounter, and all that is needed is to specialize to the case $m_{1}=m_{3}=m$ and $m_{2} \rightarrow 0$, and to harmonize the notation. He gives (his equation (4.24)) a cross section for the change $y$ in binding energy $x$. In the language of the

present paper his cross section is our differential cross section $d \sigma / d \Delta$. With $\Delta=\delta \varepsilon / \varepsilon$ we readily find that

$$
\frac{d \sigma}{d \Delta}=\frac{2 \pi}{3} \frac{\sqrt{G m a^{3}}}{V \Delta^{2}}
$$

though Heggie used units in which $G=1$ and so we have restored it in its proper place here.

This formula makes no distinction of the sign of $\Delta$ : it appears that hardening and softening encounters are equally likely. But Section 4.3 of Heggie (1975) also showed that an application of the principle of detailed balance could be used to estimate the difference in the rate of hardening and softening encounters. A similar principle, however, applies to differential cross sections, and we employ it here. From equation (A3) in Heggie \& Hut (1993), which is expressed in slightly different notation, it is easy to see that

$$
\frac{d \sigma}{d \Delta}(\Delta, a, V) a^{7 / 2} V^{2}=\frac{d \sigma}{d \Delta}\left(\Delta^{\prime}, a^{\prime}, V^{\prime}\right) a^{\prime 7 / 2} V^{\prime 2},
$$

where we have made explicit the dependence of the cross section on the initial semi-major axis of the planet and the initial speed of the passing star. On the left side we are here considering encounters which begin with values $a, V$ and end with values $a^{\prime}=a /(1+\Delta)$ and $V^{\prime}$. The right side considers information on the time-reversal of such encounters, and so $(1+\Delta)\left(1+\Delta^{\prime}\right)=1$. In the present case, with $m_{2}=0$, we have $V=V^{\prime}$. Substituting for $a^{\prime}$ and $V^{\prime}$ we deduce that

$$
\frac{d \sigma}{d \Delta}(\Delta, a, V)=(1+\Delta)^{-7 / 2} \frac{d \sigma}{d \Delta}\left(-\frac{\Delta}{1+\Delta}, a /(1+\Delta), V\right)
$$

Substituting from equation (18) we deduce that

$$
\frac{d \sigma}{d \Delta}(\Delta, a, V)=(1+\Delta)^{-3} \frac{d \sigma}{d \Delta}(-\Delta, a, V)
$$

Finally, expanding for small $|\Delta|$, we find that

$$
\frac{d \sigma}{d \Delta}(\Delta, a, V)-\frac{d \sigma}{d \Delta}(-\Delta, a, V) \simeq-3 \Delta \frac{d \sigma}{d \Delta}(\Delta, a, V) .
$$


This interesting result shows that hardening encounters are rarer than softening ones. The same qualitative conclusion results from considering non-tidal impulsive encounters, i.e. impulsive encounters in which the passing star approaches the planet or its sun to a distance less than $a$. For completeness we present here two results for this case drawn from equation (4.12) in Heggie (1975), but specialized to the present masses and translated into the notation of the present paper. They are

$$
\frac{d \sigma}{d \Delta}=\frac{8 \pi G m a}{V^{2} \Delta^{2}}\left\{\begin{array}{cc}
\left(1-\frac{4}{3 \Delta}\right), & \Delta<0 \\
\left(\frac{7}{3}+\frac{4}{3 \Delta}\right)(1+\Delta)^{-5 / 2}, & \Delta>0 .
\end{array}\right.
$$

Again we find that softening encounters have a higher cross section than hardening encounters.

Though these cross sections apply to impulsive encounters, which is a regime of relevance for soft planets, it was pointed out by Section 5.1 of Heggie (1975) that the results should be roughly applicable also for non-tidal encounters with hard systems, except for a correction due to gravitational focusing. The passing star is accelerated by the star of the planetary system until its relative velocity becomes comparable with that of the planet. In the case of stellar-mass binaries, which was the application in Heggie (1975), the outcome for hardening encounters $(\Delta>0)$ is complicated by the possible capture of the passing star. This does not occur in the case of a planetary system in the limit $m_{2} \rightarrow 0$, and so equation (23) does apply roughly (with the aforementioned correction) to planetary systems. It also helps to explain the discovery by Fregeau et al. (2006) that a planetary system of intermediate binding energy softens on average (provided that it is not so hard that capture of the passing star is energetically possible). Intermediate refers here to a planetary system in the regime $v_{c}<V<v_{\text {orb }}$, where $v_{c}$ is the critical velocity according to Fregeau et al. (2006) and $v_{\text {orb }}$ is the orbital velocity of the planet.

\subsubsection{Adiabatic encounters}

We can discuss changes of the binding energy in much the same way that we discussed changes in eccentricity in Section 2.2.2, For the parabolic case, expressions for $\Delta$ are given by Heggie (1975) and, more explicitly, by Roy \& Haddow (2003), while Heggie (2005) extends these to the hyperbolic case. Again we here give only enough to explain the form of the cross sections, and defer further detail to the Appendix.

Let $\varepsilon$ denote the binding energy of a planetary system, with semi-major axis $a=$ $G m_{1} m_{2} / 2|\varepsilon|$, and $m_{2} \ll m_{1}$. Then the relative binding energy change $\Delta=\delta \varepsilon / \varepsilon$ for an 
encounter becomes

$$
\Delta=-\sqrt{\pi} f_{1}\left(e^{\prime}\right) K^{1 / 2} \exp \left(-\frac{2}{3} K f_{2}\left(e^{\prime}\right)\right) F\left(e, \omega, \Omega, n t_{0}\right)
$$

The definition of $F\left(e, \omega, \Omega, n t_{0}\right)$ can be obtained by comparison with equation (A9). This factor is obtained from the one given by Roy \& Haddow (2003), equation (19) and Heggie (2005), equation (11) by taking out the factor $a^{2}$. Thus our function $F$ is of order unity, and depends only on the eccentricity, orientation and phase of the planetary orbit. For the purpose of our paper only an average over all possible values is of interest, and to order of magnitude we use here $F \approx 1$. Now we quote the two functions

$$
\begin{aligned}
& f_{1}\left(e^{\prime}\right)=\left(\frac{e^{\prime}+1}{2}\right)^{3 / 4} e^{\prime-2} \\
& f_{2}\left(e^{\prime}\right)=\frac{3}{2 \sqrt{2}} \frac{\sqrt{e^{\prime 2}-1}-\arccos \left(1 / e^{\prime}\right)}{\left(e^{\prime}-1\right)^{3 / 2}} .
\end{aligned}
$$

We discuss the functions for the parabolic case $e^{\prime}=1$ and for the extremely hyperbolic case $e^{\prime} \gg 1$; for the first one we have $f_{1}\left(e^{\prime}\right)=f_{2}\left(e^{\prime}\right)=1$, and in this case we reproduce the result of Roy \& Haddow (2003). In the hyperbolic limit we have asymptotically $f_{1}\left(e^{\prime}\right) \approx e^{\prime-5 / 4}$ and $f_{2}\left(e^{\prime}\right) \approx e^{-1 / 2}$, and so we get

$$
\begin{aligned}
& \Delta_{\text {par }} \approx-\sqrt{\pi} K^{1 / 2} \exp \left(-\frac{2}{3} K\right) \\
& \Delta_{\text {hyp }} \approx-\sqrt{\pi} e^{\prime-5 / 4} K^{1 / 2} \exp \left(-\frac{2}{3} K e^{\prime-1 / 2}\right) .
\end{aligned}
$$

As before we have $e^{\prime} \propto p \approx r_{p}$ for the hyperbolic case, and in both cases $K \propto r_{p}^{3 / 2}$ by definition, whence it follows that

$$
\begin{aligned}
& \Delta_{\text {par }} \propto-\sqrt{\pi} r_{p}^{3 / 4} \exp \left(-\frac{2}{3} r_{p}^{3 / 2}\right) \\
& \Delta_{\text {hyp }} \propto-\sqrt{\pi} r_{p}^{-1 / 2} \exp \left(-\frac{2}{3} r_{p}\right) .
\end{aligned}
$$

To compute differential cross sections we need to invert the function $\Delta\left(r_{p}\right)$, which can be done easily only if the exponential function dominates. In that case we have

$$
\begin{aligned}
\sigma_{\text {par }} & \propto p^{2} \propto r_{p} \propto(\ln \Delta)^{2 / 3} \\
\sigma_{\text {hyp }} & \propto p^{2} \propto r_{p}^{2} \propto(\ln \Delta)^{2}
\end{aligned}
$$

where we think of $\Delta$ as positive. Thus differential cross sections are obtained in the forms

$$
\left.\frac{d \sigma}{d \Delta}\right|_{\mathrm{par}} \propto \frac{1}{\Delta}(\ln \Delta)^{-1 / 3}
$$




$$
\left.\frac{d \sigma}{d \Delta}\right|_{\text {hyp }} \propto \frac{1}{\Delta} \ln \Delta .
$$

It is shown in Appendix $\mathrm{C}$ that, to leading order, the full results are

$$
\begin{aligned}
\left.\frac{d \sigma}{d \Delta}\right|_{\text {par }} & =\frac{2 \pi G m a}{V^{2}} \frac{1}{\Delta}(\ln \Delta)^{-1 / 3} \\
\left.\frac{d \sigma}{d \Delta}\right|_{\text {hyp }} & =\frac{\pi a^{3} V^{2}}{G m} \frac{1}{\Delta} \ln \Delta .
\end{aligned}
$$

\section{Computational Methods and Initial Model Parameters}

We use our hybrid Monte Carlo (HMC) scheme as well as direct $N$-body simulations to study the changes in the orbital elements of planetary systems induced by encounters in stellar clusters. The main limitations of the models are that all stars are single (except for the planetary companions), all have equal mass, and there is no stellar evolution. The main loss of generality is the choice of a specific range of semi-major axis for the planetary orbits. Though we argue below that the range adopted is astrophysically justified, it is one aim of the analytical work to show how the results might be generalized to values outside this range. We collect data for encounters between stars and planetary systems in different ways in the two models, as described in the following sections. The initial parameters of $N$-body and HMC models are summarized in Table 1.

\section{1. $\quad$ Direct $N$-Body models}

The N-body code we use is the publicly available NBODY6++ version. The equations of motion of each planetary system can be treated by regularization. Encounters and perturbations by flybys affect the orbital elements of these regularized pairs, and a sufficiently strong interaction with a passing star can dynamically dissolve the planetary systems. The only special refinement to mention is that we utilize the newly applied classical method with which secular errors in the integration of close binaries in stellar systems can be strongly reduced (Mikkola \& Aarseth 1998).

For all models, we adopt an isotropic Plummer model for the stars' initial phase space distribution function. This model provides a reasonable approximation for open cluster potentials. All models are in dynamical equilibrium initially. Our model units are such that $G=1, M=1, E=-0.25$, for the gravitational constant, initial total mass and

energy, respectively (standard $N$-body units, Heggie \& Mathieu (1986)). For all models, the 
individual mass of stars thus scales with $1 / N$. Planetary masses are set to the constant value of $10^{-10}$ in $N$-body units for both the $N$-body and HMC models. This choice ensures that all our planets are practically massless compared to the stellar masses. Physical units are obtained by (a) assigning an individual stellar mass $\left(m_{*}\right)$ in units of solar masses (see Table 1) to the stars and (b) defining $1 \mathrm{pc}$ as one $N$-body unit (this is the virial radius $R=G M / 4|E|)$. The length scaling law also fixes what is one $N$-body unit in $\mathrm{AU}\left(2.27 \cdot 10^{5}\right.$ for a system with solar-mass stars and 1 pc identified with one $N$-body unit).

For all runs done here all stars have equal mass, and there are no stellar binaries initially. We place one and only one planet around $N_{p}$ of the stars. For the direct $N$-body models we use 20,000 objects, $N_{p}=1000$ of which are planets, which are randomly attached to 19,000 equal mass single stars, to form a planetary system. This is a smaller particle number than in the HMC runs (see below). Other properties, such as the initial Plummer model, and the absence of tidal fields, are exactly as in the case of Monte Carlo models.

In all of our $N$-body runs except two (runs E, EH) all planets have the same initial eccentricity $e_{0}$, and we do different runs with differing values of $e_{0}$ (Models 1-6: see Table 1). The eccentricities of our models range from 0.01 (in model 1) to 0.99 (in model 6). The use of several different models with different eccentricities has two effects: (i) it improves the statistical data for the relatively small particle number (and relatively small number of scattering events) in the direct $N$-body simulations; (ii) it allows us to consider the dependence of scattering statistics on the planet's orbital eccentricity, and to facilitate a more straightforward comparison with analytic models (Heggie \& Rasio 1996, Roy \& Haddow 2003, Heggie 2005). Runs E and EH, however, cover all initial eccentricities using a thermal eccentricity distribution $f(e)=2 e$, which is also adopted in the HMC models. For small $e$ 's, this distribution approximates the Rayleigh distribution which is expected as a consequence of dynamical instability and relaxation (Zhou et al. 2007). In all calculations, the initial phase and orientation of the planets' orbits, including the direction of their angular momentum vector and their periastron, are randomized in the obvious senses.

The semi major axes of the planets are chosen with a logarithmic distribution, i.e. a constant $d N_{p} / d \log a$, between $3-50 \mathrm{AU}$ for run E and between $0.03-5 \mathrm{AU}$ for run EH. The initial distribution of semi-major axes in the runs E and EH corresponds to the soft and hard planetary systems in the HMC runs, respectively. Note that we denote these planetary systems as soft and hard to distinguish between the two types of runs (with physically wider and closer planetary systems). We would like to remind the reader again here that our definition of hard and soft differs from the dynamical definition of Fregeau et al. (2006). According to the dynamical definition a planetary system is hard if $V<0.75 v_{c}$, where $v_{c}$ is the critical velocity of the three-body system. We choose the orbital velocity 
$v_{\text {orb }}$ of the planetary system as parameter to distinguish encounters. If we use for the average $V^{2}$ in our models twice the cluster velocity dispersion, we get in $N$-body units $V^{2} / v_{\text {orb }}^{2}=N\left(a_{\mathrm{AU}} / 2.27 \cdot 10^{5}\right)$, where we have again taken one $N$-body length unit to be $1 \mathrm{pc}$ and $a_{A U}$ is the planet's semi major axes in astronomical units. In Table 1 we give the value of $V / v_{\text {orb }}$ for our initial planetary systems.

In choosing these parameters we have been guided by theoretical and observational considerations. The range of 3-50 AU of semi major axis represents the location where gas giant planets are most likely to form (Ida \& Lin 2005). Migration due to protoplanet-disk interaction may repopulate the regions interior to 3 AU (Lin, Bodenheimer \& Richardson 1996) and dynamical instabilities could eject planets beyond 50 AU (Lin \& Ida 1997). But most of the gas giant planets may remain near the location of their formation. While gas giants are most likely to have formed with nearly circular orbits, dynamical instabilities could excite their eccentricities to the point of ejection.

Up to now, one or a few Jupiter-mass planets, with periods less than a few years, have been found around less than $10 \%$ of the nearby solar-type stars (Marcy et al. 2005), though planets with longer periods are expected to be more common (Trilling et al. 1998, Armitage et al. 2002, Cumming et al. 2007). The total mass of planets is too small to significantly perturb the internal dynamics of any stellar cluster. Thus, for the simulations to be presented here, we include $N_{p}$ planets with very small masses of order $10^{-10}$, such that the mass ratio of planetary to average stellar mass is $1.9 \cdot 10^{-6}$, comparable to the mass of terrestrial planets. The total and individual mass of our planets is small enough, that they do not contribute any significant dynamical feedback to the cluster of $N_{s}$ stars.

As for the stellar system itself, our aim has been to represent a typical rich young star cluster such as the Orion region. However, most stars are formed in binary and multiple systems. In stellar clusters, the presence of binary stars can significantly speed up the relaxation process due to their larger mass (Gao et al. 1991). They also strongly enhance the frequency of close three- and four-body encounters due to their larger cross section. This will also affect planetary systems (Laughlin \& Adams 1998), and the influence of interactions with binary stars on planetary systems will be investigated in future work. 
Table 1: Initial parameters of $N$-body and HMC models ${ }^{\text {a }}$

\begin{tabular}{cc|cccccc}
\hline \hline Model & $N_{*}$ & $m_{*}\left(M_{\odot}\right)$ & $N_{p}$ & $a(\mathrm{AU})$ & $e_{0}$ & $V / v_{\text {orb }}{ }^{\mathrm{b}}$ & Type \\
\hline 1 & $1.9 \cdot 10^{4}$ & 1 & $10^{3}$ & $3-50$ & 0.01 & $0.49-2.05$ & $N$-body \\
2 & $1.9 \cdot 10^{4}$ & 1 & $10^{3}$ & $3-50$ & 0.1 & $0.49-2.05$ & $N$-body \\
3 & $1.9 \cdot 10^{4}$ & 1 & $10^{3}$ & $3-50$ & 0.3 & $0.49-2.05$ & $N$-body \\
4 & $1.9 \cdot 10^{4}$ & 1 & $10^{3}$ & $3-50$ & 0.6 & $0.49-2.05$ & $N$-body \\
5 & $1.9 \cdot 10^{4}$ & 1 & $10^{3}$ & $3-50$ & 0.9 & $0.49-2.05$ & $N$-body \\
6 & $1.9 \cdot 10^{4}$ & 1 & $10^{3}$ & $3-50$ & 0.99 & $0.49-2.05$ & $N$-body \\
$\mathrm{E}$ & $1.9 \cdot 10^{4}$ & 1 & $10^{3}$ & $3-50$ & $f(e)=2 e$ & $0.49-2.05$ & $N$-body \\
$\mathrm{EH}$ & $1.9 \cdot 10^{4}$ & 1 & $10^{3}$ & $0.03-5$ & $f(e)=2 e$ & $0.05-0.65$ & $N$-body \\
Soft & $3.0 \cdot 10^{5}$ & 1 & $3.0 \cdot 10^{4}$ & $3-50$ & $f(e)=2 e$ & $1.98-8.13$ & HMC \\
Hard & $3.0 \cdot 10^{5}$ & 1 & $3.0 \cdot 10^{4}$ & $0.03-5$ & $f(e)=2 e$ & $0.20-2.57$ & HMC
\end{tabular}

${ }^{\text {a }} N_{*}$ is the total initial number of stars and planetary systems, $m_{*}\left(M_{\odot}\right)$ is the mass of one star in solar units, $N_{p}$ is the initial number of planetary systems, $a(\mathrm{AU})$ is the initial range of the semi-major axes in $\mathrm{AU}$, and $e_{0}$ is the initial eccentricity. b we use $V / v_{\text {orb }}=\sqrt{N\left(a_{\mathrm{AU}} / 2.27 \cdot 10^{5}\right)}$ to obtain an approximate range of values, cf. Section 3.1.

There is one important technical aspect of these $N$-body runs that remains to be described. While direct $N$-body models contain less intrinsic approximations than other simplified models, such as the HMC model, for our purposes there is a drawback, because it is very difficult to identify isolated two-body encounters in an $N$-body model. In fact it has been discussed, whether a real $N$-body system's relaxation process can be described by the standard model of uncorrelated small angle two-body encounters between individual stars (Theuns 1996). Generally, it is possible to identify an encounter by checking the minimum distance to the closest neighbour of any given particle, to get the $r_{p}$ and the velocity $V_{p}$ at closest distance. However, it is very difficult to determine the proper initial parameters of an encounter, because the scintillation and fluctuation of the $N$-body potential perturbs any orbit even at moderate distances. Despite all these factors it is possible operationally to determine encounter event data, similar to that and to be compared with the HMC model. In fixed time intervals of one $N$-body time unit (approximately one half-mass initial crossing time) we monitor orbital elements of all planetary systems $(e, a)$. If any one of them has changed by more than $5 \cdot 10^{-7}$ since the previous time, we assume an encounter took place. We measure $\delta e$ and $\Delta$, and thus have a data bank of encounters for the $N$-body system similarly to that for the HMC runs. From a theoretical point of view there may be uncertainties about this procedure, but we don't know any better alternatives, and judging from 
the results it seems to be a reasonable operational procedure. The value of the hyperbolic eccentricity $e^{\prime}$ cannot be determined a priori; however from comparison with analytical models (see figures below) one can see that the deduced values of $e^{\prime}$ lie in a completely reasonable range.

\subsection{Hybrid Monte Carlo method}

We use the hybrid Monte Carlo (HMC) method developed by Spurzem \& Giersz (1996), Giersz \& Spurzem $(2000,2003)$ to model the evolution of a star cluster with a large number of stars and planetary systems. The latter are considered as if they were a binary; binaries are treated with the Monte Carlo scheme to follow their relaxation with each other and with single stars in the cluster, while single stars are described by the anisotropic gaseous model based on the Fokker-Planck approximation (Louis \& Spurzem 1991). Close encounters between planetary systems and a single star are followed as in the cited papers using a direct few-body integrator employing regularization methods (cf. e.g. Mikkola 1997). For HMC the planet mass in N-body units is very small, $6.33 E-12$ and the mass ratio of planets to stars is $1.9 \mathrm{E}-6$ also, as in the case of $N$-body runs, and consistent with the notion that our planets have a mass comparable to terrestrial planets.

We describe results of two sets of models, each with 300,000 single stars, 30,000 of which initially have a planet (see Table 1). The semi-major axes of planetary systems introduce another independent scale into the otherwise scale-free $N$-body system. We have chosen initially to keep the planetary scale $a$ (semimajor axes) constant relative to the scale radius of the stellar system, independently of $N$. As a consequence the size of planetary systems measured in $N$-body units is the same for HMC and $N$-body models. Note that this means the squared orbital velocity of planets scales as $1 / N$, where $N$ is the particle number. In terms of cluster velocity dispersion our planetary systems become weaker (softer) with larger $N$. We will discuss the scaling behaviour of encounters between planetary systems and single stars in more detail in sections 5.3 and 5.4.

All parameter choices for the planetary systems are analogous to those of the $N$-body simulations, as explained before, and some more details are given in the table. For each set of parameters, two models with independently generated initial phase space distributions are adopted to boost the statistical significance of our results. The runs were continued for approximately five initial half-mass crossing times. This is a small interval for relaxation, but already enough to sample interactions between planetary systems and single stars appropriately. The time step of the HMC code is small enough to resolve these very accurately

in time, because it is given by the gaseous model code for the single star component. Despite 
of small time steps it is taken care that every binary is treated by the Monte Carlo procedure for relaxation with the correct rate and no over-relaxation occurs. A detailed description of this and the initial setup for encounters is given in Giersz \& Spurzem (2003). Here, we will only summarize the main points of the setup procedure. Whether a planetary system actually suffers from an encounter with a star is determined randomly for each time-step and planetary system. First, a maximum impact parameter is chosen to cover all physically interesting cases, $p_{\max }=2000$ AU. From $p_{\max }$ we determine an encounter rate $\dot{N}$ using the simple prescription $\dot{N}=n A v_{\text {rel }}$, where $n, A=\pi p_{\text {max }}^{2}, v_{\text {rel }}$ are the local planetary number density, the geometrical cross section corresponding to $p_{\max }$, and the actual relative velocity of the star and planetary system chosen for an encounter. In every time step we determine whether an encounter takes place by comparing the probability obtained from the encounter rate with a random number. After we have identified an encounter we pick an actual impact parameter $p$, from a random distribution in $p^{2}$. Using this with energy and momentum conservation we transform the encounter from the cluster frame into the interaction frame and start the actual direct integration of the encounter using packages from Aarseth's NBODY6 code. After the termination of the direct integration of the encounter the new parameters of the planetary system are computed and the outcome of the encounter is identified: flyby, dissolution or exchange and transformed from the interaction frame into the cluster frame.

\section{Results of numerical experiments}

The three parts of this section summarize our numerical results and their interpretation. We begin with a discussion of the destruction of planetary systems in stellar encounters, and the escape of the resulting population of freely-floating planets. Then we give a largely empirical description of the results of the non-destructive encounters. This analysis paves the way for the final subsection, in which we interpret the results as directly as possible in terms of differential cross sections.

We note here that many of our encounters lead to very small changes of the eccentricity or semi-major axis of the planetary system (see some of the following plots). The changes are often sufficiently small as to cast doubt on the significance of such a result. Numerical errors are introduced in the three-body integration for HMC and by the stochastic background noise of potential fluctuations which are present in the direct $N$-body simulations. To distinguish broadly those encounters which might be regarded as unreliable from the remaining results, we define certain criteria, namely $K<80$ and $|\Delta|>5 \cdot 10^{-7}$, which help to identify the most robust results. In some of the following interpretative plots we show results from the full set

of encounters, and in other cases only a limited subset. The selection of the representative 
Table 2: Summary of results of hybrid Monte Carlo (HMC) runs

\begin{tabular}{|l|rrrr|}
\hline \hline Model & HMC soft 1 & 2 & HMC hard 1 & 2 \\
\hline$N_{\text {events }}$ & 1995262 & 2426598 & 3406206 & 3650990 \\
$t_{N-\text { body }}$ & 15.28 & 15.68 & 20.67 & 30.31 \\
$\tau_{\text {cr }}=t / t_{\mathrm{cr}, 0}$ & 5.40 & 5.54 & 7.30 & 10.71 \\
$\tau_{\mathrm{rh}}=t / t_{\mathrm{rh}, 0}$ & $5.93 \mathrm{E}-03$ & $6.08 \mathrm{E}-03$ & $8.02 \mathrm{E}-03$ & $1.18 \mathrm{E}-02$ \\
$N_{\mathrm{pl}-\text { diss }}$ & 149 & 162 & 7 & 9 \\
$N_{\mathrm{pl}-\text { diss-esc }}$ & 10 & 10 & 3 & 3 \\
$N_{\mathrm{pl}-\mathrm{ff}}$ & 139 & 152 & 4 & 6 \\
$N_{\mathrm{pl}-\mathrm{ff}} / \tau_{\mathrm{rh}}$ & 23440 & 25000 & 498.1 & 508.5 \\
$N_{\mathrm{pl}-\mathrm{ff}} / \tau_{\mathrm{cr}}$ & 25.74 & 27.44 & 0.548 & 0.560 \\
$x_{\mathrm{pl}-\mathrm{ff}, \mathrm{rh}}$ & 0.7813 & 0.8333 & 0.0166 & 0.0170 \\
$x_{\mathrm{pl}-\mathrm{ff}, \mathrm{cr}}$ & $8.58 \mathrm{E}-04$ & $9.15 \mathrm{E}-04$ & $1.83 \mathrm{E}-05$ & $1.87 \mathrm{E}-05$ \\
Scaled $x_{\mathrm{pl}-\mathrm{ff}, \mathrm{rh}}$ & 0.0673 & 0.0718 & $1.43 \mathrm{E}-03$ & $1.46 \mathrm{E}-03$ \\
\hline
\end{tabular}

$N_{\text {events }}$ is the number of interactions with planetary systems, $t_{N \text {-body }}$ is the time of termination of the simulation in $N$-body units, $t_{c r, 0}$ is the initial crossing time, $t_{r h, 0}$ is the initial half-mass relaxation time, $N_{\mathrm{pl} \text {-diss }}$ is the number of dissolved planetary systems, $N_{\text {pl-diss-esc }}$ is the number of planets escaped from the system after the dissolution of the planetary system, $N_{\mathrm{pl}-\mathrm{ff}}$ is the number of "freely floating" planets.

The quantities $x_{\mathrm{pl}-\mathrm{ff}, \mathrm{rh}}$ and $x_{\mathrm{pl}-\mathrm{ff}, \mathrm{cr}}$ denote the probability for one planet to become a free floater, and are just obtained from the previous two lines by dividing by the total number of planets. $x_{\mathrm{pl}-\mathrm{ff}, \mathrm{cr}}$ can be directly compared with $N$-body results below, compare Eq. 34. $x_{\mathrm{pl}-\mathrm{ff}, \mathrm{rh}}$ will scale with the two-body relaxation time and therefore as $N_{2} \ln \left(\gamma N_{1}\right) / N_{1} \ln \left(\gamma N_{2}\right)$, with $N_{1}=300.000$ (HMC run), $N_{2}=19.000$ ( $N$-body run),

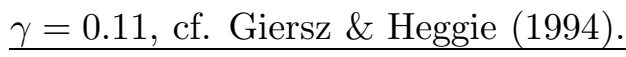

data will be clearly stated in the respective paragraphs or figure captions.

\subsection{Dissolution of planetary systems}

First, we examine the overall statistics on planetary-system retention. In Table 2 we provide some basic data which are generated with the Monte Carlo (HMC) scheme. The simulations were stopped after a few million encounters had taken place between planets and passing stars. The actual evolutionary duration corresponds to some 5-10 initial half-mass crossing times. The table summarizes some interesting information on the dissolution of planetary systems and the creation of free floaters in the HMC models. For comparison, we also present in Table 3, the analogous data which are generated with the $N$-body scheme. 
Table 3: Summary of results of direct $N$-body runs

\begin{tabular}{|c|c|c|c|c|c|c|c|c|}
\hline Model & 1 & 2 & 3 & 4 & 5 & 6 & $\bar{E}$ & $\overline{\mathrm{EH}}$ \\
\hline$N_{\text {events }}$ & 24338 & 45071 & 52060 & 70151 & 86951 & 81655 & 62022 & 6263 \\
\hline$t_{N \text {-body }}$ & 72.0 & 130. & 153. & 170. & 181. & 166. & 134. & 200. \\
\hline$\tau_{\text {cr }}$ & 25.5 & 46.0 & 54.1 & 60.1 & 64.0 & 58.7 & 47.4 & 70.7 \\
\hline$\tau_{\mathrm{rh}}$ & 0.32 & 0.59 & 0.69 & 0.77 & 0.81 & 0.75 & 0.60 & 0.9 \\
\hline$N_{\text {pl-diss }}$ & 33 & 66 & 69 & 89 & 63 & 63 & 70 & 2 \\
\hline$N_{\text {pl-diss-esc }}$ & 3 & 5 & 4 & 5 & 8 & 8 & 3 & 0 \\
\hline$N_{\mathrm{pl}-\mathrm{ff}}$ & 30 & 61 & 65 & 84 & 55 & 55 & 67 & 2 \\
\hline$N_{\mathrm{pl}-\mathrm{ff}} / \tau_{\mathrm{rh}}$ & 93.8 & 103.4 & 94.2 & 109.1 & 67.9 & 73.3 & 111.7 & 2.22 \\
\hline$N_{\mathrm{pl}-\mathrm{ff}} / \tau_{\mathrm{cr}}$ & 1.18 & 1.33 & 1.20 & 1.39 & 0.85 & 0.94 & 1.41 & 0.028 \\
\hline$x_{\mathrm{pl}-\mathrm{ff}, \mathrm{cr}}$ & $1.18 \mathrm{E}-03$ & $1.33 \mathrm{E}-03$ & $1.20 \mathrm{E}-03$ & $1.39 \mathrm{E}-03$ & $8.5 \mathrm{E}-04$ & $9.4 \mathrm{E}-04$ & $1.41 \mathrm{E}-03$ & $2.80 \mathrm{E}-05$ \\
\hline$x_{\mathrm{pl}-\mathrm{ff}, \mathrm{rh}}$ & 0.0938 & 0.103 & 0.0942 & 0.109 & 0.0679 & 0.0733 & 0.112 & $2.22 \mathrm{E}-03$ \\
\hline$\xi_{\mathrm{pl}-\mathrm{ff}, \mathrm{cr}}$ & 1.35 & 1.48 & 1.35 & 1.57 & 0.98 & 1.05 & 1.61 & 1.54 \\
\hline$\xi_{\mathrm{pl}-\mathrm{ff}, \mathrm{rh}}$ & 1.33 & 1.50 & 1.35 & 1.57 & 0.96 & 1.06 & 1.59 & 1.51 \\
\hline
\end{tabular}

All quantities have the same meaning as in Table 2. The last two lines give $\xi$, defined as the ratio of $x$ obtained from the $N$-body model divided by the average of the two corresponding HMC results, i.e. $\xi_{\mathrm{pl}-\mathrm{ff}, \mathrm{cr}}=x_{\mathrm{pl}-\mathrm{ff}, \mathrm{cr}, \mathrm{Nbody}} / x_{\mathrm{pl}-\mathrm{ff}, \mathrm{cr}, \mathrm{HMC}, \mathrm{av}}$ and $\xi_{\mathrm{pl}-\mathrm{ff}, \mathrm{rh}}=x_{\mathrm{pl}-\mathrm{ff}, \mathrm{rh}, \mathrm{Nbody}} / x_{\mathrm{pl}-\mathrm{ff}, \mathrm{rh}, \mathrm{HMC}, \mathrm{av}, \mathrm{scaled}}$.

For all models, we provide, in the tables, the rates of free floater liberation, alternatively in terms of the cluster's crossing or relaxation time. In the models which represent a population of soft planets, we create about one free floater per crossing time and about 100 per relaxation time ( $N$-body). In contrast to previous claims (Smith \& Bonnell 2001), only very few planets escape during the whole simulation - one order of magnitude less than there are free floaters, even though the initial orbital velocity of typical planets is larger than the velocity dispersion of the cluster.

In order to rule out the possibility that the retention of disrupted planets in the shallow potential of the host cluster may be due to an under-representation of close encounters by the HMC scheme, an analogous model is simulated with the $N$-body scheme. In order to compare the rates of free floater and escaper creation between these two approaches, which were obtained for practical and technical reasons with different numbers of particles and planetary systems, we first have to apply an appropriate scaling factor. In our system of $N$-body units (used here for both methods) the total mass is constant (unity) and individual stellar masses scale as $1 / N$. Rates per crossing time scale only by the number of planets, because in $N$-body units the cross section, if gravitational focusing prevails, scales inversely proportional to $N$ and the stellar number density is proportional to $N$. However, in the comparisons between the rates per relaxation time we should additionally scale by the ratio 
of half-mass relaxation times for the two models. The exact scaling expression is given in the caption of Table 2 .

The tables show that both the rates per crossing time per planet, and the (scaled) rates per relaxation time agree well, but the numbers differ, in the sense that the rate of free floater creation is larger by some $35 \%$ in the $N$-body system. However, the intrinsic variation of $N$-body results for different eccentricities is as large as that, therefore we conclude that our HMC and $N$-body results do agree reasonably with each other. The $N$-body result is that approximately 100 dissolutions of planetary systems occur per relaxation time in this case, regardless of their initial eccentricity. Contrary to the standard expectation that highly eccentric planetary systems should be more prone to disruption (Hurley \& Shara 2002), we find that the disruption rate is essentially insensitive to the planets' original eccentricity. In fact, there is a slight tendency for fewer disruptions, in the case where all planetary systems are initialized with $e=0.99$.

In order to understand this puzzling result, we have examined the encounter activity between planetary systems and stars in the $N$-body models. We find that, for highly eccentric planetary systems, there is indeed more encounter activity (e.g. more KS terminations) by some $40 \%$ (as compared to models with modest eccentricities, e.g. 0.6). Nevertheless, these encounters do not necessarily lead to free-floaters. After a brief episode of temporary liberation (KS termination), some of the highly eccentric planets quickly become attached to intruding stars. The exchange of the host stars occurs as a consequence of three- or more-body interaction. The many-body effects may also contribute to the small residual differences in the number of disrupting planetary systems between the HMC and $N$-body simulations because, in general, the many-body effects should increase the dissolution rate (as seen in the $N$-body simulations). This difference vanishes for the simulations of stellar encounters with hard planetary systems because the stellar cluster and the planetary systems are dynamically well segregated and the many-body effect should play a lesser role. While this effect is potentially very interesting, it is not in the scope of this paper to study it in further detail.

\subsection{Semi major axis and eccentricity changes}

With the aid of analytic formulae, we now analyse the consequences of stellar encounters including the vast majority which did not lead to the dissolution of the planetary systems. The main objective in this section is to determine the cross sections for eccentricity and semi major axis changes. 


\subsubsection{Domains of encounter classes}

In the analysis of the numerical data, it is useful to make direct comparison with the analytic results in $\S 2$. Following the prescriptions in $\S 2$ (Heggie 2005), we first show the location of the limited set of encounters in the normalized relative velocity-impact parameter plane for the hard and soft planetary systems (Figs. 1 and 2). In these figures, the ordinate is $V / \sqrt{G M_{123} / a}$, while the abscissa is given by $r_{p} / a$ for each encounter. The parameter space is separated by three domains in accordance with the nature of the encounters. The vertical line $\left(r_{p}=a\right)$ separates very close interactions from tidal encounters. The line separating adiabatic from non-adiabatic encounters is defined by $V / r_{p}=v_{c} / a$, where $v_{c}=\sqrt{G M_{12} / a}$ is the circular velocity of the planet. The line which separates near-parabolic from hyperbolic encounters is given by the condition $e^{\prime}=2$.

Figure. 11 represents a model for the hard planetary systems. Only a small number of encounters (for small $r_{p}$ ) with hard planetary systems are non-adiabatic. There is a considerable number of near-parabolic encounters for $r_{p}>a$. In contrast, the cloud of representative points shifts upward in the model for the soft planetary systems (Fig. 2). This systematic difference is due to the planets' larger semi-major axes. Consequently, there are many non-adiabatic encounters with hyperbolic speeds and $r_{p}>a$ for the soft planetary systems. The number of near-parabolic encounters is negligible. Very small changes in $|\Delta|\left(<5 \cdot 10^{-7}\right)$ may be affected by numerical errors. The omission of these potentially spurious points accounts for the absence of points in the lower right corner of the figures. The condition that $K<80$ also truncates the distribution of data points on the right hand side. A corresponding set of figures cannot be constructed for the $N$-body simulations due to the lack of complete dynamical information. In these simulations the magnitude of $v$ for individual encounters is not well determined.

\subsubsection{Correlated changes of orbital elements}

We now analyze the correlations between the relative change in the planet's energy and the magnitude of $\delta e$. Figures. 3 and 4 illustrate the full set of encounters for the soft planetary systems in the HMC and $N$-body models (run E), respectively. Here we can clearly identify a huge number of encounters with very small changes. The data points on the upper right hand corner of these figures represent planetary systems with modest changes in both the relative energy and eccentricity. These figures show that there is a correlation between the relative semi-major axis and eccentricity changes.

This correlation is much weaker for the bulk of encounters with very small changes, 


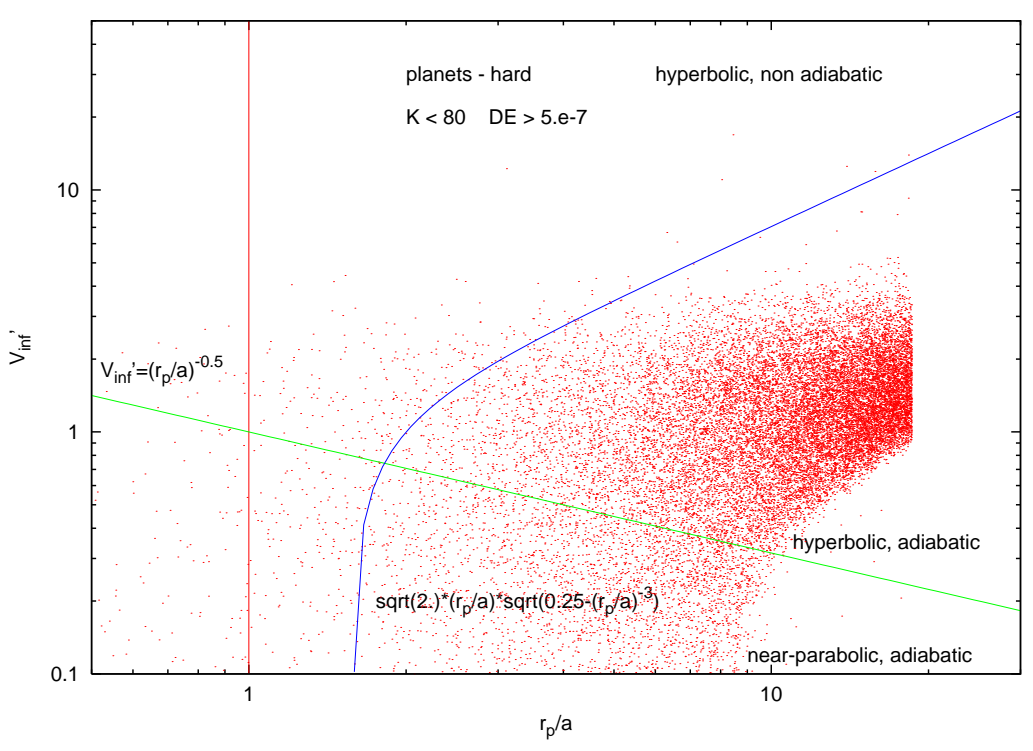

Fig. 1. - Location of encounters for hard planetary systems in the hybrid Monte Carlo model, plotted according to the scaled velocity at infinity and the minimum distance $r_{p}$ in units of the semi-major axis $a$ of the planetary system. Solid lines indicate boundaries between encounters which are close or wide, adiabatic or non-adiabatic, hyperbolic or nearparabolic. All details of scaling and definition of the boundaries are given in the main text: note that they are analogous to those in Fig. 1 of Heggie 2005.

which is one of the reasons we consider changes at this level may be due to spurious random noise. For the $N$-body models, the uncorrelated data representing very small orbital changes may also be due to the unresolved potential fluctuations and multiple concurrent encounters (see discussion above on how we sample the encounter data in the $\mathrm{N}$-body model). In the HMC model, where the maximum impact parameter in the simulation was set to be 2000 AU, even the weakest encounters are well defined. The uncorrelated measurement of energy and eccentricity changes represents the small limits of numerical accuracy in the few-body integration and in their initialisation.

As in the $N$-body data, however, for the data analysis we only use those encounters which obey $|\Delta|,|\delta e|<5 \cdot 10^{-7}$ to exclude numerically unreliable results (see discussion in Sec. 3.1). Both figures show that the changes are very symmetric with respect to their sign, i.e. there are as many cases with eccentricity decrease as with eccentricity increase. Note that for the system with larger $N$ (the HMC models have 300,000 stars, as compared to the $N$-body models, which had only 19,000), the median values of $\Delta$ and $\delta e$ are roughly one order of magnitude smaller. This difference is consistent with the interpretation that 


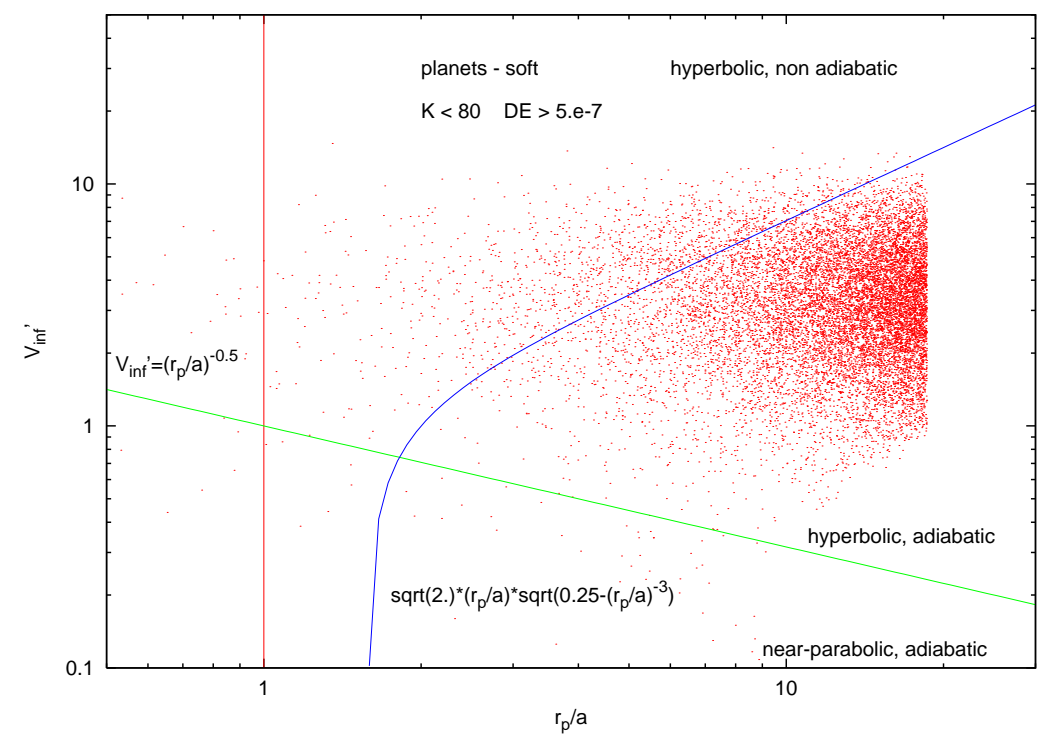

Fig. 2.- Same as Fig. 1, but for soft planetary systems.

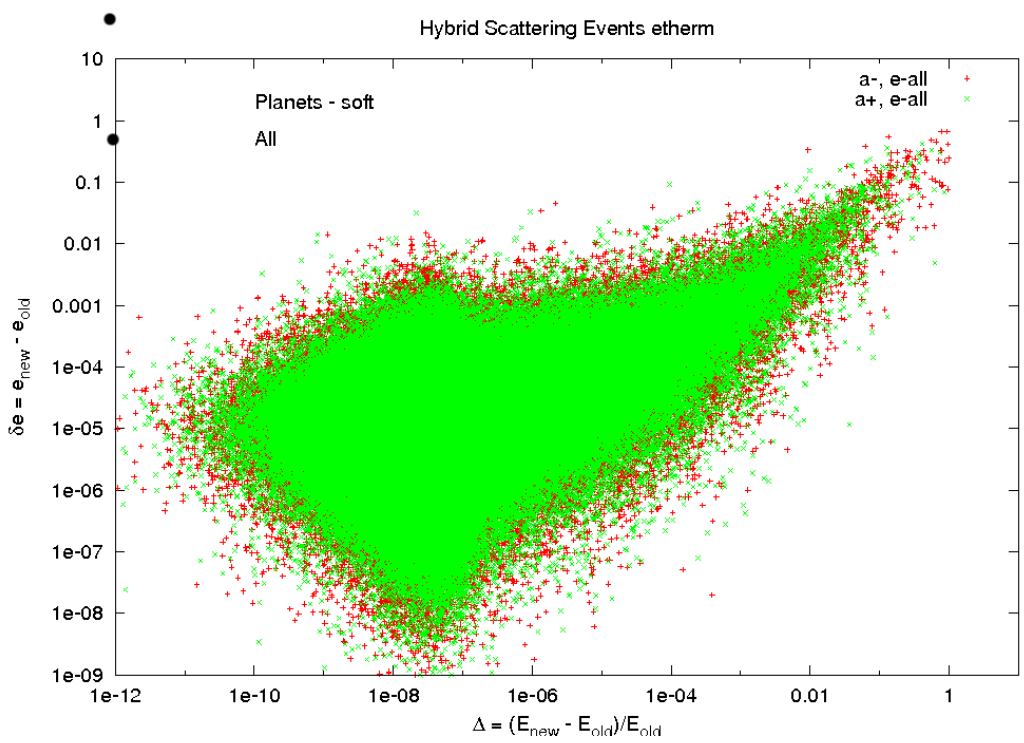

Fig. 3.- Relative energy change vs. eccentricity change for soft planetary systems in the hybrid Monte Carlo model. All encounters are included.

encounters become weaker for larger systems. 


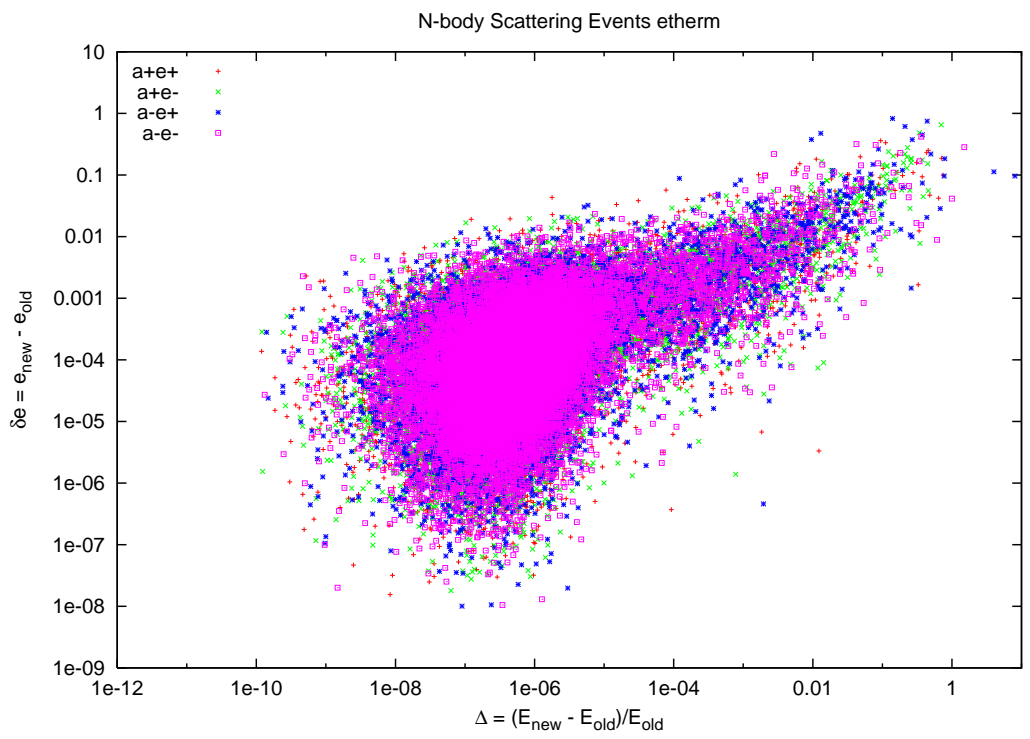

Fig. 4.- As Fig. 3, but for the direct $N$-body model (soft-planetary systems) with an initially thermal eccentricity distribution.

\subsubsection{Parabolic versus hyperbolic encounters}

The next three Figs. 5, 66 and 7 again illustrate the dynamical changes in the plane defined by $\Delta$ and $\delta e$. The main purpose of this analysis is to show the dependence of the outcome on the initial relative speeds between the encountering stars. Indeed it was the study of these results which forced us to extend theoretical results in the literature to the case of hyperbolic encounters.

For the HMC model (Fig. [5) we show three sets of encounters, depicted by different colours of points: $e^{\prime}<2$ (red), $10<e^{\prime}<30$ (green), and $e^{\prime}>50$ (blue); the general limits defined above $\left(K<80, \Delta>5 \times 10^{-7}\right)$ were also applied. These three groups of data points represent nearly parabolic, intermediate, and hyperbolic encounters. Note that most hyperbolic encounters lead to very small changes in $a$ and $e$. Only the parabolic encounters satisfy the necessary condition for planetary disruption and major orbital element changes, i.e. $|\Delta|>1$ and $\delta e \sim 1$ respectively.

For the $N$-body model (Figs. 6 and 7), although the encounters cannot be individually distinguished by their $e^{\prime}$, the overall results agree with those obtained from the HMC model. (Here data for very small changes, i.e. with $\delta e<5 \times 10^{-6}$, are omitted, see figure). Figure 6 shows $N$-body results with an initially thermal eccentricity distribution, while Fig. 7 presents results from run 1 (with $e_{0}=0.01$ initially for all planetary systems). 


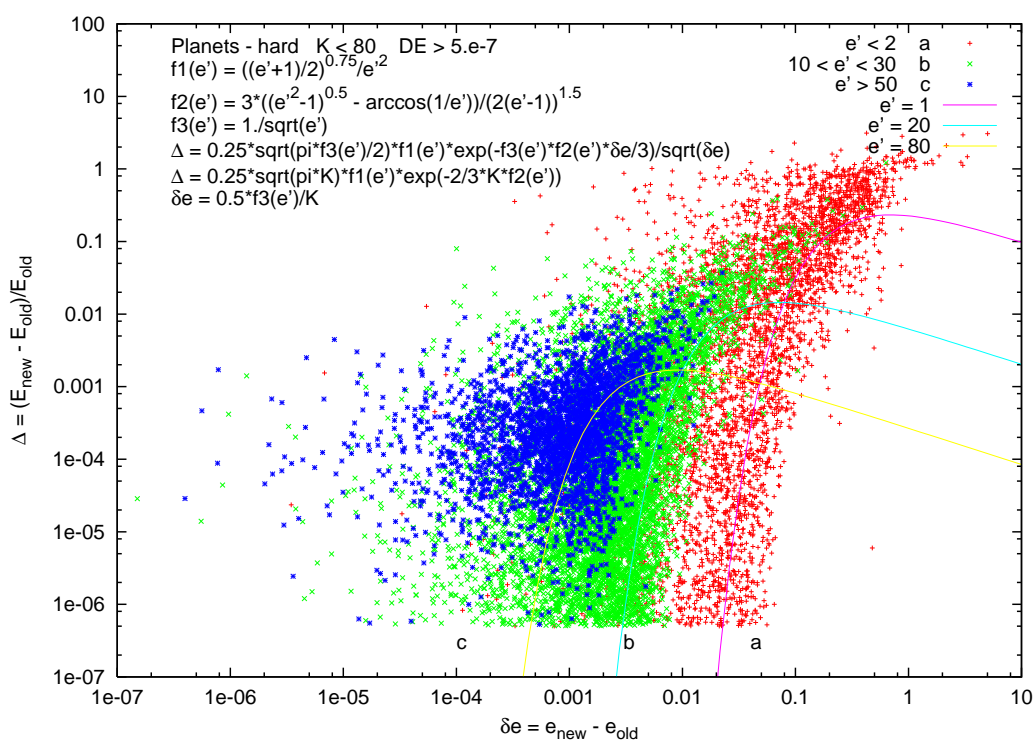

Fig. 5.- Location of encounters for hard planetary systems in the hybrid Monte Carlo model, plotted by relative energy change and eccentricity change, for three cases with different $e^{\prime}$ (near-parabolic, intermediate, and extremely hyperbolic; see main text for more details). For comparison solid lines are plotted from analytic expressions of Heggie \& Rasio (1996) and Heggie (2005).

In order to compare these numerical data with the analytic results (see $\S 2$ ), we super impose in all three figures, the correlated magnitude of $\delta e$ and $\Delta$ derived from the analytic expressions in equations (12) and (24), for three different values of $e^{\prime}=1,20,80$, respectively. The results of the HMC simulations and those of the $N$-body model E (with an initial thermal eccentricity distribution), i.e. Figs. 5 and 6, agree fairly well with each other and with theory. Both models indicate that planets break up primarily through parabolic encounters. Note that $e^{\prime}$ increases from right to left in all three figures. It can also be seen that the encounters which have been excluded (due to small changes or large $K$ ) are extremely hyperbolic.

\subsubsection{Planetary systems with nearly circular initial orbits}

This case requires separate discussion. The plot of the $N$-body results for $e_{0}=0.01$ (nearly circular orbits, Fig. 7) shows some differences from the previous two cases. For planetary systems which suffer large orbital changes, the correlation between $\delta e$ and $\Delta$ is much more pronounced, with less scattering of the points at the upper right hand side of the figure. For a given $\delta e$ these encounters for nearly circular binaries tend to exhibit smaller 


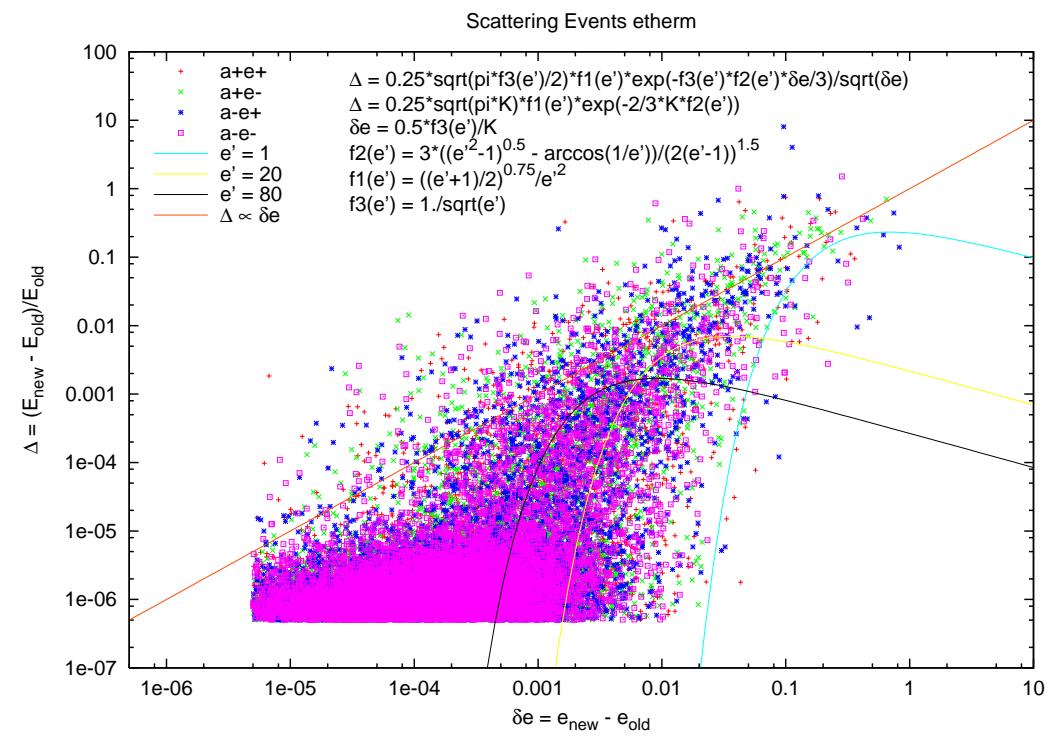

Fig. 6.- Location of encounters for planetary systems with an initially thermal eccentricity distribution in the direct $N$-body model (soft-planetary systems), plotted by relative energy change and eccentricity change. For comparison the same solid lines as in Fig. 5 are plotted, based on analytic expressions of Heggie \& Rasio (1996) and Heggie (2005).

energy changes than in the previous cases.

Now we turn to a theoretical interpretation of these differences. Several theoretical results change when one considers strictly circular initial orbits (cf. Appendix A.1). This can also be seen in Fig. 7, where we have plotted curves based on assuming the same dependence of $\delta e$ and $\Delta$ on $e^{\prime}$ as for non-circular orbits. This comparison is unsatisfactory, particularly for the small values of $K$, which correspond to the limit of strong encounters.

First consider the encounters for which $|\delta e| \gg e=0.01$. In this case analytical theory for a circular binary is relevant. In eqs.(A4) and (A5), which give the change in eccentricity for this case, the main dependence on the distance of closest approach is in the exponential factors. The same exponential dependence occurs in the formula for the relative energy change in the parabolic case (eq.(A10)) and in the hyperbolic case (not shown). Therefore we may expect $\Delta \propto \delta e$ for large changes in eccentricity (and energy). The dashed line in Fig, 7 has the same slope, and it is followed by the trend of the points for large $\delta e$ and $\Delta$.

There is a further complication, because the figure we are discussing deals with soft planetary systems, and the closest encounters are distinctly non-adiabatic (Fig.21), and indeed impulsive. To find out how $\delta e$ and $\Delta$ are correlated in this case we may make use of eqs.(5), 
(6), from which it can be deduced that

$$
\delta\left(e^{2}\right)=\left(1-e^{2}\right)\left\{-\frac{\delta E}{E}-\frac{\delta\left(J^{2}\right)}{J^{2}}\right\} .
$$

Now for an impulsive encounter with a circular binary $\delta E=\mathbf{v} . \delta \mathbf{v}$ and $\delta J^{2}=2 a^{2} \mathbf{v} . \delta \mathbf{v}$. But we also have $J^{2}=G M a$ in this case, where $M$ is the total mass of the binary, and a quick calculation shows that the lowest order contribution to $\delta\left(e^{2}\right)$ vanishes. (It is obvious that it must do, as this contribution would be proportional to $\mathbf{v} . \delta \mathbf{v}$, and would give both positive and negative values, whereas we must have $\delta\left(e^{2}\right)>0$ for an initially circular binary.) It follows that $\delta\left(e^{2}\right)$ must be proportional to $|\delta \mathbf{v}|^{2}$, in this case, and so $\delta e \propto|\delta \mathbf{v}|$. But $\delta E$ is also proportional to $|\delta \mathbf{v}|$, as mentioned in Sec2.2.1, and so again we deduce that $\Delta \propto \delta e$.

In this case of impulsive encounters, it was mentioned in Sec.2.3.1 that $\Delta \sim D \equiv \delta\left(e^{2}\right)$. This suggests a steepening of the trend in the dependence of $\Delta$ on $\delta e$ at the extreme right of the diagram, and this is not observed.

In the remainder of the diagram $(|\delta e| \ll 0.01)$ the binary essentially behaves as one with a non-zero initial eccentricity, and the distribution of points may be expected to behave much as in the two previous cases. The analytical expressions for $\delta e$ and $\Delta$, i.e. eqs. (A3) and (A9), are approximately proportional to $e$ for small $e$, and so the curves in this diagram should be scaled by a factor of order 0.01. This brings them into better accord with the points corresponding to more distant encounters. The numerical results indicate that the amplitude of eccentricity change is considerably larger than that of the change in energy or semi major axis. Since the dissolution rate is essentially independent of the initial eccentricity, planets which formed with circular orbits can quickly become eccentric.

\subsubsection{Dependence on the intruding star's distance of closest approach}

In $\S 2$, we show that the magnitude of a planet's dynamical response to a stellar encounter depends on both the impact speed (through $e^{\prime}$ ) and the distance of closest approach (through $K)$. We now interpret our numerical results in terms of the dependence of $K$ given by the analytic expressions. In Figure 8 the dynamical changes in the planetary orbits are depicted as in the three figures before, but this time plotted in the plane of $K$ and $\Delta$. Only HMC simulations are represented, for $N$-body models it was impossible to obtain reliable encounter parameters for each interaction and compute $K$ (see Sec. 3.1). This plot can be compared with the analytic results more directly because the latter are often expressed as functions of $K$, whereas the correlation with $\delta e$ is more indirect.

The numerical results obtained with the HMC scheme (Fig. 8) agree very well with 


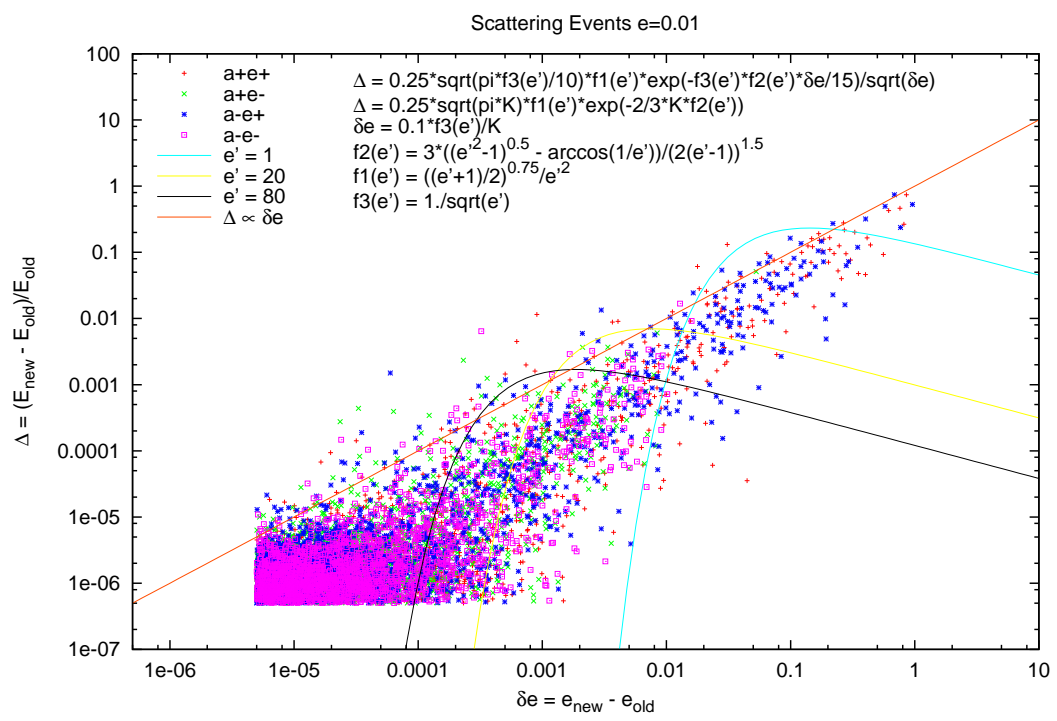

Fig. 7.- Location of encounters for planetary systems with initial eccentricity $e_{0}=0.01$ in the direct $N$-body model for soft planetary systems, plotted by relative energy change and eccentricity change. Again the same solid lines as in Fig. 5 are plotted from analytic expressions of Heggie \& Rasio (1996), Roy \& Haddow (2003) and Heggie (2005).

the analytic expression. In this case, the fractional energy change at large $K$ is correlated with $e^{\prime}$. This trend indicates that in the high $K$ limit, a vast majority of encounters are very hyperbolic. Only nearly parabolic $\left(e^{\prime}<2\right)$ and non adiabatic, close (small $\left.K\right)$ stellar encounters lead to planets' disruption, i.e. $|\Delta|>1$. In addition, it follows from Fig. 5 that most non adiabatic, close encounters lead to major eccentricity changes, i.e. $|\delta e| \sim 1$.

\subsection{Rates of energy and eccentricity evolution}

\subsubsection{Distributions of $\delta e$ and $\Delta$}

For another comparison of the HMC and NBODY6++ results we have just binned the changes of $\Delta$ and $\delta e$ and computed the numbers $N(\Delta), N(\delta e)$ normalized to the total number of events. Figs. 9 and 10 show the results as a function of $\delta e$ and $\Delta$, respectively. Note that this is an unnormalized measure of the total cross sections. In the following section we will deduce properly the normalized differential cross sections as well.

Here, we first observe that the shapes of the encounter frequency distributions are similar, albeit they are shifted (the numbers for the HMC model are smaller by about one 


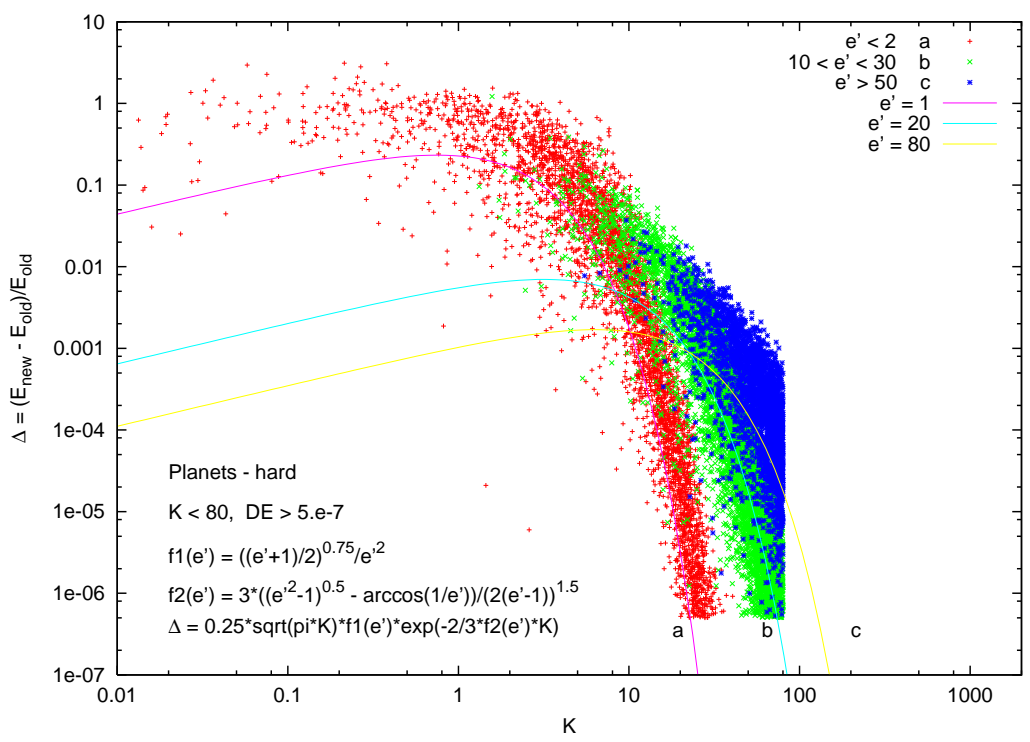

Fig. 8.- Location of encounters for hard planetary systems in the hybrid Monte Carlo model, plotted by relative energy change as a function of $K=\left(r_{p} / a\right)^{3 / 2}$, for three cases with different $e^{\prime}$ (near-parabolic, intermediate, extremely hyperbolic; see main text for more details). For comparison solid lines based on analytic expressions of Heggie (2005) are plotted.

order of magnitude, except for small $|\delta e|)$. There are two possible explanations for this shift, and both effects may contribute. With a larger particle number in the HMC model all changes are on average about an order of magnitude smaller than in the $N$-body model (compare discussion of Fig. 3) and so the curves are shifted to the left. On the other hand, in the $N$-body system individual encounters with extremely small changes (which are easily observed in the HMC model) are obscured by the background of stochastic potential fluctuations.

The nearly logarithmic distribution $N(\delta e) \propto \delta e^{-1}$ for $\delta e>10^{-4}$ implies that the mean square changes of eccentricity are dominated by close encounters and large $\delta e$. We also note here that, in the HMC models we observe that the initially thermal eccentricity distribution is preserved, as would be expected.

Turning now to the distribution of $\Delta$, we note that, for energy changes with $\Delta \gtrsim 0.1$, negative changes of $\Delta$ are more probable, i.e. that there is a preferred trend towards softening of planetary orbits. This confirms the discovery by Fregeau et al. (2006) that there is a range of intermediate planetary orbits with $v_{c}<V<v_{\text {orb }}$ for which there is a net surplus of softening encounters as compared to hardening ones. We also confirm this with our analytical results, as discussed qualitatively at the end of Section 2.3.1, and the result is also visible in 


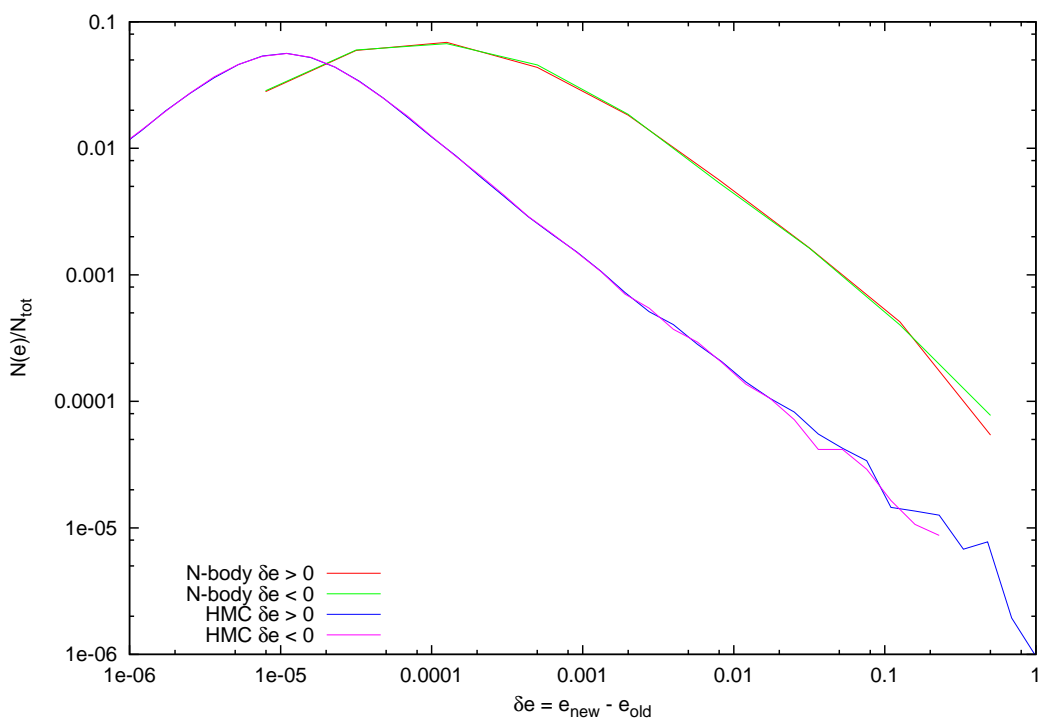

Fig. 9.- Fractional number of encounters as a function of eccentricity change (soft planetary systems), compared between the hybrid Monte Carlo and $N$-body models with an initially thermal eccentricity distribution. The data are given separately for positive and negative changes.

Fig. 13 (differential cross sections) with better statistical quality.

\subsubsection{Normalization for differential cross sections}

Finally we compute properly normalized differential cross sections, and compare them with theory. We restrict the numerical data to those from the HMC runs. Differential cross sections can be obtained from our numerical results using the binned data $N(\Delta)$ described previously. Because the cross sections depend on $a$ and $V$, however (Section 2), some postprocessing is necessary.

We define $P$ to be the probability that a given encounter results in a value of $\Delta$ which lies within a certain bin. Then

$$
P=\frac{1}{\pi p_{\max }^{2}} \frac{d \sigma}{d \Delta}(\Delta, a, V) \delta \Delta,
$$

where $\delta \Delta$ is the range of the bin. Let us suppose that the differential cross section has a power-law dependence on $a$ and $V$, i.e.

$$
\frac{d \sigma}{d \Delta}(\Delta, a, V)=\frac{d \sigma}{d \Delta}(\Delta, 1,1) a^{\alpha} V^{\beta}
$$




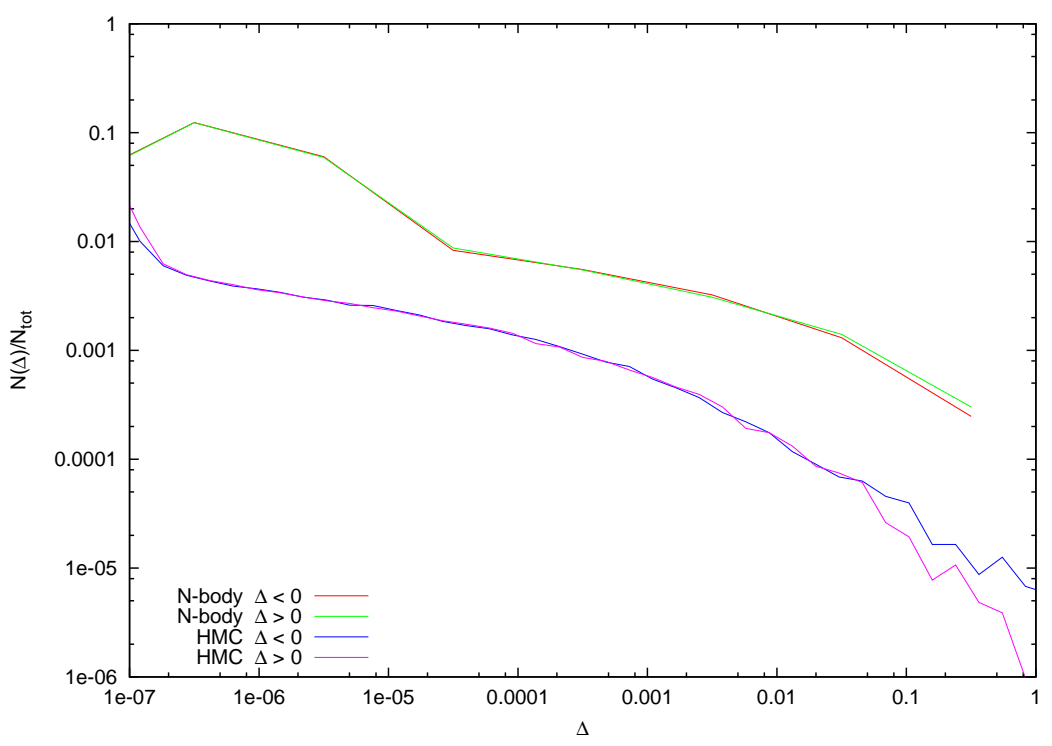

Fig. 10.- Fractional number of encounters as a function of relative energy change (soft planetary systems), compared between the hybrid Monte Carlo and $N$-body models with an initially thermal eccentricity distribution. The data are given separately for positive and negative changes.

where $\alpha, \beta$ are certain constants and $d \sigma / d \Delta(\Delta, 1,1)$ is the normalization factor. Then, equating $N(\Delta)$ to its expected value, we have

$$
\frac{N(\Delta)}{\delta \Delta}=\frac{d \sigma}{d \Delta}(\Delta, 1,1) \sum_{\text {encounters }} \frac{a^{\alpha} V^{\beta}}{\pi p_{\max }^{2}} .
$$

In the following four figures, the theoretical cross sections from Section 2 are scaled with the summation factor on the right side of this equation, and plotted, along with the numerical data, against $\Delta$ (or $D=\delta\left(e^{2}\right)$ ). Note that the summation factor has to be computed separately for each theoretical expression.

Exactly the same prescription can be applied to determine the cross section for eccentricity changes. The resulting differential cross sections are given in Figs. 11 and 12 for hard and soft planets, respectively. For comparison with analytic expressions, we also plot in these figures a number of theoretical results from Section 2.2. normalized as in equation (33). 


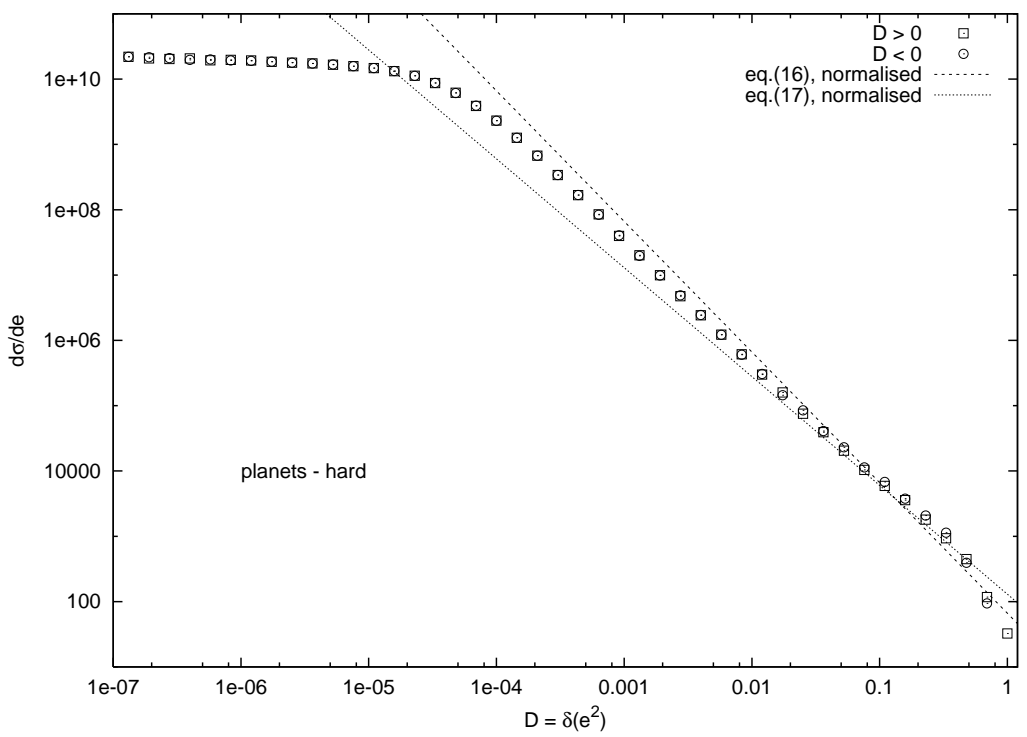

Fig. 11. - Differential cross section for eccentricity changes of hard planets, as a function of eccentricity change (strictly, $D=\delta\left(e^{2}\right)$ ) for the hybrid Monte Carlo model. The analytic results obtained by formulae from Section 2 are plotted as straight lines, while the binned numerical results appear as points; see detailed explanation in the main text.

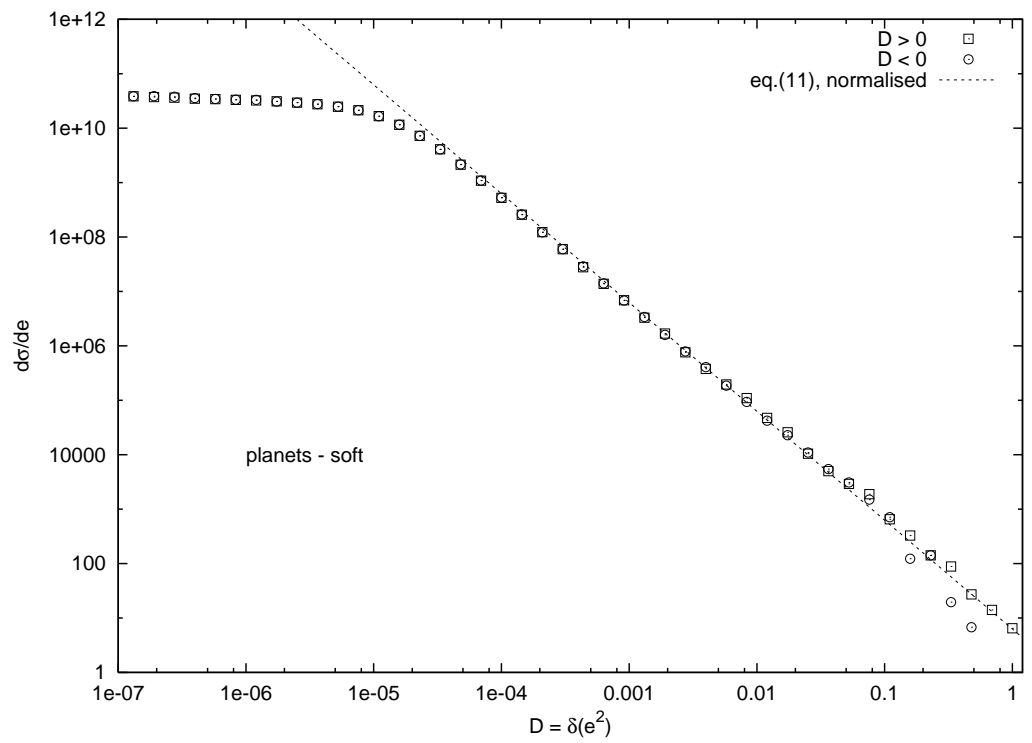

Fig. 12.- Same as Fig. 11, but for soft planetary systems. 


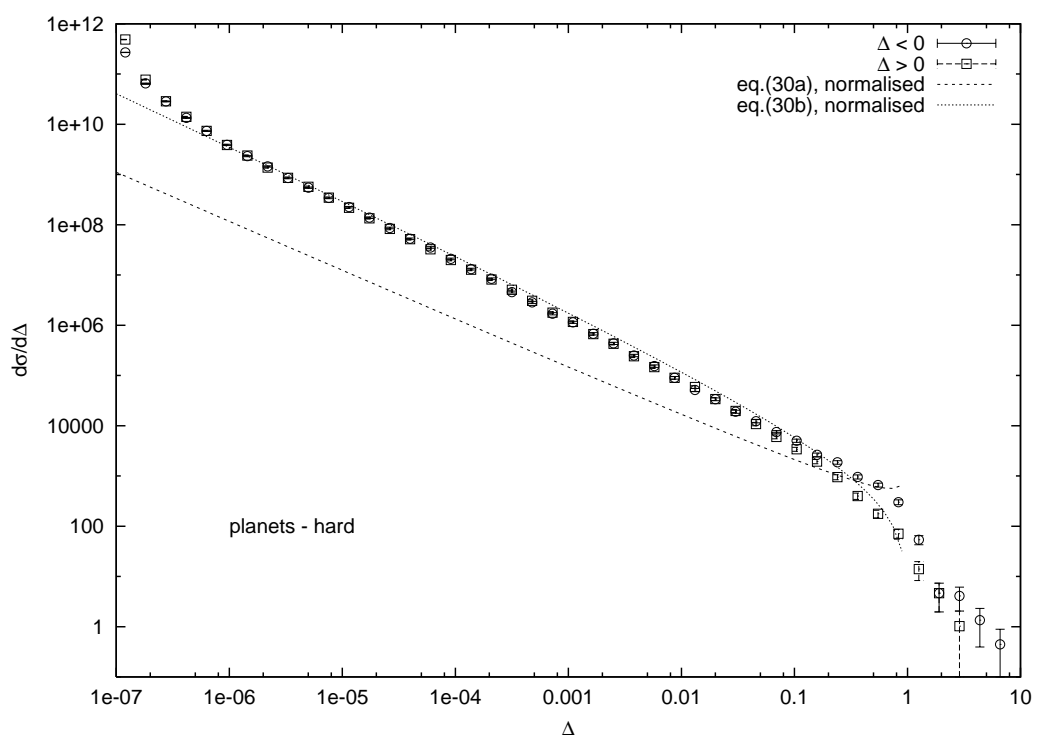

Fig. 13. - Scaled differential cross section for relative energy changes of hard planets, as a function of relative energy change for hybrid Monte Carlo model. The analytic results obtained by formulae from Section 2 are plotted as lines, while the binned numerical results appear as points, see detailed explanation in the main text.

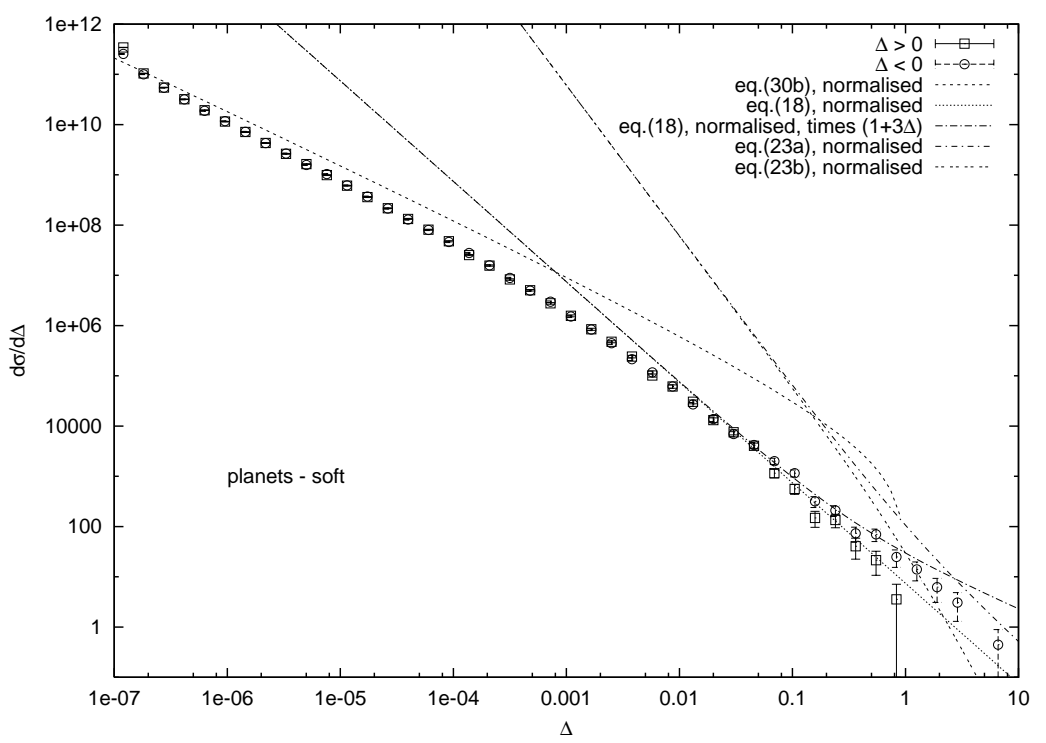

Fig. 14. - Same as Fig. 13, but for soft planetary systems.

\subsubsection{Differential cross section for eccentricity evolution}

We first consider the case of hard planets (Fig.11). The two theoretical cross sections in the figures correspond to the limits of parabolic (dotted) and extremely hyperbolic (dashed) 
encounters. The former correspond to closer encounters and therefore larger values of $|D|$. The crossover between the two formulae should occur at $D \simeq 0.3\left(\frac{V^{2} a}{G m}\right)^{3 / 2}$. The trend of points with $|D| \gtrsim 10^{-4}$ indeed steepens appropriately as $|D|$ decreases below about 0.01 . However, since there is a considerable range in the semi-major axis, this figure merely indicates that the range over which the change of slope takes place is reasonable. Figure 1 shows that there is a large overlap (in $r_{p} / a$ ) of parabolic and hyperbolic encounters, and so the position of the data points between the two theoretical lines is acceptable. The notable exception is the strong flattening below $|D| \simeq 10^{-4}$. This saturation results, however, from the chosen value of $p_{\max }$.

Next, we turn to the case of soft planets (Fig. 12), where the interpretation, in terms of tidal impulsive encounters, is more straightforward. The plotted analytical result (equation (11)) is satisfactory down to values below $D \sim 10^{-4}$. The saturation value in the small $D$ limit is entirely consistent with that expected, using equation (8), for encounters at a distance $r_{p} \sim p_{\max }$.

\subsubsection{Differential cross section for fractional energy change}

Differential cross sections for the relative energy changes are displayed in Figs. 13 and 14. Here it is the case of hard planetary systems which is most straightforward. Almost all of the data agrees very well with the analytic expression for hyperbolic adiabatic encounters (equation (30) ). The apparent cutoff at $|\Delta|=1$ is spurious, since the validity of the formula is restricted to large values of $|\ln | \Delta||$ (or $|\Delta|<<1$. Figure 1 indicates that the closest encounters should be nearly parabolic, and so we have also plotted the corresponding theoretical result (dashed in Fig. 13). It is not clear whether it plays a significant role here. We do notice, however, that softening encounters (with $\Delta<0$ ) have a higher cross section than hardening encounters, in accordance with the qualitative discussion at the end of Section 2.3.1.

We attribute the rise in the numerical cross section at small $|\Delta|$ in Figure 13 to numerical errors. For encounters with $p \lesssim p_{\max }$, we estimate from equations (26) and (2) that $\Delta \sim$ $10^{-44}$ (where even the exponent is an order-of-magnitude estimate!). This magnitude is much smaller than numerical errors, and even much smaller than the start-up error of initiating the three-body integration at a finite distance of order $p_{\max }$. Such encounters thus have errors which place them at the left hand side of this figure.

The interpretation of the cross section for energy changes in soft systems (Fig. 14) is 
the most complicated of all. It is also most relevant since dissolution occurs more frequently in this limit. At the extreme left of this figure, the result of numerical errors is again visible. Over a range of small values of $|\Delta|$, the numerical results lie just below the theoretical curve for adiabatic hyperbolic encounters (equation (30) ). This $|\Delta|$ dependence intersects a steeper curve which represents the impulsive tidal encounters (equation (18)). The numerical results follow the steepening trend. By comparing the two equations we expect the crossover

to occur at $|\Delta \ln | \Delta|| \simeq \frac{2 \pi}{3}\left(\frac{G m}{a V^{2}}\right)^{3 / 2}$. Though most of the encounters with "soft" planets give values of $V^{2} a /(G m)$ above 1, the median value is only of order 3 (see Fig. 2), and so the crossover is expected to occur at values of $|\Delta|$ no less than about 0.1 . It seems likely that the interpretation of the data is complicated by a considerable admixture of encounters which really should be classified as hard.

For relatively large values of $|\Delta| \gtrsim 0.1$, we differentiate positive and negative energy changes (see equation (22) ) by multiplying the value given in equation (18) with a factor $(1+3|\Delta|)$ (Fig. 14). In magnitude this corresponds quite well to the separation in the data points between those for $\Delta>0$ (circles) from those for $\Delta<0$ (squares).

Also shown for soft planets are the two differential cross sections for impulsive nontidal encounters (equations (23) ). Though they again display the difference between positive and negative values of $\Delta$, it is not clear what quantitative role they play in relation to this data. We presume that this is due to the fact that the systems are not really very soft. By comparing equations (23) and (18), we estimate that the crossover should occur at $\Delta \simeq \frac{16 \sqrt{G m}}{V \sqrt{a}}$. It is only for considerably softer systems that one would expect to see this crossover.

\section{Summary and Discussion}

\subsection{Methodological Aspects}

Very few planets are found in old open and globular clusters; we have explored the scenario that this deficiency is associated with disruption of planetary systems in long-lived star clusters due to stellar encounters. Freely floating planets observed in star forming regions support our scenario. We have studied two prototype systems with direct $N$-body (NBODY) and hybrid Monte Carlo (HMC) simulations of star clusters which contain a large number of planetary systems. The direct $N$-body model represents a cluster similar to the Hyades with 20,000 stars and 1000 planets, while the HMC models represent a centrally concentrated globular cluster such as 47 Tuc with 300,000 stars and 30,000 planets. We 
have recorded changes of planetary orbits due to encounters and the creation of free floaters (remaining in the cluster) and escapers. Our main results are summarized in Tables 2, 3, We have shown that there is a consistency between analytic and numerical models in terms of the cross section of encounters between planetary systems and a single star, and, to first order, the two models give consistent results for the liberation rates of planets (creation of free floaters). Encounters have been measured and recorded in large numbers and compared with analytic estimates for the changes of semi-major axis and eccentricity. It turns out that the majority of encounters in our simulations are well approximated by the analytic results, which corresponds to the fact that the encounters are either adiabatic (hyperbolic or parabolic) or impulsive and tidal in nature. In the adiabatic regimes, evolution of the planetary orbital elements is a diffusive process and proceeds in both directions, i.e. with similar probabilities in eccentricity excitation and damping as well as semi major axis increases and declines. However, there is also a non-negligible number of encounters which are not adiabatic and lead to relatively small minimum distances. These close encounters give stronger changes in the orbital parameters of planetary systems, and in particular a net injection of orbital energy and increase in the semi major axis.

Two fundamentally different numerical methods are used in this series of investigations. They are the direct $N$-body and Hybrid Monte Carlo algorithms, the latter being based on the Fokker-Planck equation for the single stars. Both schemes provide consistent results with each other and the analytic results (see figures and Tables). Remaining differences e.g. in the rates of liberation of planets per crossing time, are attributable to the more complex dynamical nature of the $N$-body system - for many encounters with larger impact parameters the assumption that they are uncorrelated isolated two-body encounters is no longer fully justified. The examination of how well diffusion coefficients for individual twobody encounters are reproduced in direct $N$-body systems is, however, beyond the scope of this paper. Interested readers are referred to discussions elsewhere (Theuns 1996).

\subsection{Application to real clusters or associations}

In order to allow the reader to extract more specific information from our general results, we are presenting some data regarding the free floaters in our simulations, and a scaling procedure to estimate the corresponding results for some real clusters.

In the following Table 4 we provide the timescale $\tau_{\text {ff }}$ for a planet to become a free floater, by using data of Table 2, averaged between the two physically equivalent runs). We obtain $\tau_{\mathrm{ff}}$ by the definition $\tau_{\mathrm{ff}}=\tau_{\mathrm{cr}} / x_{\mathrm{pl}-\mathrm{Ff}, \mathrm{cr}}\left(\right.$ or $\tau_{\mathrm{ff}}=\tau_{\mathrm{rh}} / x_{\mathrm{pl}-\mathrm{ff}, \mathrm{rh}}$, which should give an equivalent result except for roundoff errors). Table 4 provides exemplary values for $\tau_{\text {ff }} / \tau_{\text {cr }}$ and $\tau_{\text {ff }} / \tau_{\text {rh }}$, 
Table 4: Time Scale $\tau_{\mathrm{ff}}$ for the liberation of free floaters relative to $\tau_{\mathrm{cr}}$ and $\tau_{\mathrm{rh}}$

\begin{tabular}{c|rrrrr}
\hline Model & $\tau_{\text {ff }} / \tau_{\text {cr }}$ & $\tau_{\text {ff }} / \tau_{\text {rh }}$ & $\tau_{\text {ff }}$ Gyrs $($ Hya) & $\tau_{\text {ff }}$ Gyrs $($ ONC) & $\tau_{\text {ff }}$ Gyrs $(47$ Tuc $)$ \\
HMC soft 1 & 1128.0 & 1.239 & 15.79 & 0.367 & 0.92 \\
HMC hard 1 & 54054. & 59.52 & 756.8 & 17.57 & 44.27
\end{tabular}

respectively, which in fact are just the inverse averaged $x$-values taken from Table 2 .

To determine values of $\tau_{\mathrm{ff}}$ in physical units we must employ the scaling of $\tau_{\mathrm{ff}}$ with cluster parameters. If we assume planetary semi-major axes to stay constant in model units, as was done in the scaling between HMC and $N$-body models here, $\tau_{\mathrm{ff}}$ is independent of $N$, just as $x_{\mathrm{pl}-\mathrm{ff}, \mathrm{cr}}$. So if a typical half-mass crossing time can be deduced from observational data for some real clusters (as we will do below for the Orion Nebula and the Hyades clusters) we can just directly compute $\tau_{\mathrm{ff}}$ from that and our measured value of $x_{\mathrm{pl}-\mathrm{ff}, \mathrm{cr}}$. If, however, a value of the half-mass relaxation time is more well known from observations (as in the case of the globular cluster 47 Tuc), we have to be aware that $x_{\mathrm{pl}-\mathrm{ff}, \mathrm{rh}}$ scales with the relaxation time (see explanations after Table 2).

The data to obtain lifetimes of planetary systems in physical units are obtained as follows: The globular cluster 47 Tuc is a typical old stellar cluster with a very high central density (of order $10^{6} M_{\odot} / \mathrm{pc}^{3}$ ). We expect this cluster to be the most hostile environment for planetary systems. From the Harris catalog (Harris 1996) we get the following data for 47 Tuc: $r_{h}=2.79^{\prime}$, with a distance of $R=4.5 \mathrm{kpc}$, which implies that $r_{h}=3.6 \mathrm{pc}$; the half-mass relaxation time is given as $\log t_{\mathrm{rh}}=9.48$, i.e. $t_{\mathrm{rh}}=3.02$ Gyrs. In order to apply our model, we need to determine the particle number, or total mass, which are poorly constrained in the literature. Therefore we use the following procedure, which is sufficient for an order-ofmagnitude approach. We adopt the relaxation time scaling of $t_{\mathrm{rh}} \propto N r_{h}^{3 / 2} / \log (\gamma N)$. From our HMC model, with $N=300.000, r_{h}=0.77 \mathrm{pc}$, and $t_{\mathrm{rh}}=7.28 \cdot 10^{7} \mathrm{yrs}$, we then deduce a particle number of $N=1.4 \cdot 10^{6}$ stars for 47 Tuc. The scaling factor for the relaxation time between our HMC model cluster and 47 Tuc is 4.06 . Hence we predict a time scale $\tau_{\mathrm{ff}}$ to be 0.305 (14.66) relaxation times for a planet to become a free floater, i.e. 0.92 (44.27) Gyrs. These results have been obtained from our finding that $\tau_{\mathrm{ff}} / t_{\mathrm{rh}}$ scales inversely with the relaxation time, because in systems with larger $N$ more planets are liberated per relaxation time). The values outside and inside brackets are for the soft and hard planetary systems respectively. In other words, we predict for 47 Tuc, the dissolution rate is all $(7 \%)$ of soft (hard) planetary systems per relaxation time. We have said that the hard time scale is 14.66 relaxation times (44.27 Gyrs, based on Table 4); here "soft" refers to planets with semi-major axes between 3 and 50 AU, while "hard" refers to 0.03 to 5 AU, both in logarithmically equal distribution. Fregeau et al. (2006) quote a lifetime of 10 Gyrs for hard planetary systems 
$(0.05 \mathrm{AU})$ in their statistical analysis, which is also in qualitative agreement with earlier work of Smith \& Bonnell (2001). From these previous papers, one would have expected that most present-day planetary systems in globular clusters have been dissolved. While the given time scales are only a rough estimate, detailed models as presented in this paper and also in Fregeau et al. (2006) suggest that a significant fraction of planets with semi-major axes between 0.03 and $5 \mathrm{AU}$ (hard in our notation, intermediate in that of Fregeau et al. (2006) ) could have survived stellar encounters in this cluster (see further discussions below).

The Orion nebula cluster (ONC) is an example of a young star cluster with intermediate central density, of order $2 \cdot 10^{4} M_{\odot} / \mathrm{pc}^{3}$. For this system, we use a half-mass radius of $0.8 \mathrm{pc}$ and a velocity dispersion of $2.34 \mathrm{~km} / \mathrm{s}$, to obtain the half-mass crossing time as $3.25 \cdot 10^{5}$ yrs. For ONC also the half-mass relaxation time $\left(t_{\mathrm{rh}}=6.5 \cdot 10^{6} \mathrm{yrs}\right)$ and particle number $N=2800$ are provided (Hillenbrand \& Hartmann 1998). Planetary system lifetimes are then obtained by using our measured lifetimes in units of crossing times (see Table 4).

Note that a scaling using the measured half-mass relaxation time of the ONC would provide approximately the same results, if the proper Coulomb scaling factor is applied to our measured time scales (in units of relaxation time, see Tables 2, 3).

For the Hyades cluster, which is a relatively low density open cluster we use the halfmass radius $r_{h}=4.5 \mathrm{pc}$ and central velocity dispersion of $0.3 \mathrm{~km} / \mathrm{s}$ to determine a half-mass crossing time of approx. $1.4 \cdot 10^{7}$ yrs (Perryman et al. 1998). Again we derive physical timescales in Table 4. Note that these estimates are subject to many uncertainties, such as the use in our models of stars of equal mass.

\subsection{Total Cross Sections and Timescales to get Free Floaters}

Fregeau et al. (2006) provide in their Monte Carlo simulations cross sections for orbital changes of planetary systems obtained from a large set of simulations of three-body encounters. We want to compare their results with ours for the case of ionization of planetary systems due to relatively close encounters. For that we use the usual ansatz for the planetary system lifetime as

$$
\tau_{\mathrm{ff}}=\frac{1}{n \sigma_{\text {ion }} V}
$$

where $n=\rho / m$ is the average particle density, $\sigma_{\text {ion }}$ is the total cross section for dissolution of planetary systems, and $V$ is the typical encounter relative velocity. The relevant total cross section for ionization in our case is taken from equation (23) for non-tidal impulsive 
encounters as

$$
\begin{aligned}
\sigma_{\text {ion }} & =\frac{8 \pi G m}{V^{2}} a\left[F\left(\Delta_{\max }\right)-F\left(\Delta_{\min }\right)\right] \\
F(\Delta) & =\frac{1}{\Delta}\left(\frac{2}{3 \Delta}-1\right)
\end{aligned}
$$

where we have obtained the total cross section for "ionization" by integrating equation (23) $(\Delta<0)$ from some suitable $\Delta_{\min }$ to $\Delta_{\max }$. With $\Delta_{\max } \rightarrow-\infty$ and the assumption that $\Delta_{\min }=-1$ (the minimum required relative energy change for ionization is unity) we get $F\left(\Delta_{\max }\right)=11 / 3$, thus

$$
\sigma_{\text {ion }}=\frac{40 \pi}{3} \frac{G m}{V^{2}} a
$$

and

$$
\tau_{\mathrm{ff}}=\frac{3 V}{40 \pi G \rho a}
$$

This result is in agreement with Fregeau et al. (2006) in the case of equal stellar masses, although we get for the case of 47 Tuc slightly larger values of the planetary lifetimes. Possible reasons are that the distribution of encounter velocities in a real cluster differs from those in three-body experiments, and that planets, which just escape from a three-body encounter with a very small velocity at infinity could easily be recaptured by their own host star or another star due to potential fluctuations.

Note that the lifetime of planetary systems as given in equation (37) does not vary with the particle number $N$, provided the planetary system's semi-major axes remain constant relative to the $N$-body scale (system scaling radius), consistent with our numerical results. If one is interested in ionisation time scales relative to the relaxation time we have to look at the quantity

$$
\frac{1}{x_{\mathrm{pl}-\mathrm{ff}, \mathrm{rh}}}=\frac{\tau_{\mathrm{ff}}}{\tau_{\mathrm{rh}}} \sim \frac{G m \log \Lambda}{V^{2} a}
$$

where $\log \Lambda$ is the Coulomb logarithm contained in the relaxation time. If we take the standard Coulomb $\operatorname{logarithm} \log \Lambda=\log (\gamma N)$, with $\gamma=0.11$ (cf. Giersz \& Heggie (1994)) we get for the scaling

$$
x_{\mathrm{pl}-\mathrm{ff}, \mathrm{rh}}=\frac{\tau_{\mathrm{rh}}}{\tau_{\mathrm{ff}}} \sim \frac{V^{2} a}{G m \log (\gamma N)} \sim \frac{N}{\log (\gamma N)}
$$

This is indeed the empirically measured scaling behaviour, as we have commented in the caption to Table 2. Note that in these considerations, also in the choice of initial conditions for our runs with different particle number (HMC and $N$-body) we have neglected the possible influence of a separate variation of the planetary orbit scale $a$ with respect to the $N$-body 
system's scaling radius $R$. Since $\tau_{\mathrm{ff}} \sim R / a$ the effect would be easy to estimate to first order (if only the size of planetary orbits relative to the cluster size is varied). If however, the scale changes are due to different cluster models (e.g. King models of different concentration) further numerical studies would be required.

\subsection{Discussion}

We have provided a further step towards a self-consistent modelling of the origin of differences in the frequency of planetary systems between star clusters and the galactic field due to encounters of planetary systems with passing stars. For two representative star clusters with particle numbers resembling open and globular clusters, respectively, we have tested analytical cross sections against two different series of self-consistent numerical simulations under realistic conditions of a star cluster (not merely a series of three-body scattering experiments). For the illustration of our results, we have provided liberation time scales for planets (to become a free floater) in their respective cluster environment. In contrast to previous work we have extended our study to planetary systems ranging from 0.05 AU to $50 \mathrm{AU}$ in semi-major axis and all eccentricities. To ease discussion and understanding we divide our planetary systems in two classes (hard and soft), and determine the average time scale for their survival, see Table 4. We find that the liberation rate of planets per crossing time is constant, and per relaxation time scales with the scaling factor $N / \log \Lambda$, where $\log \Lambda$ is a Coulomb logarithm (provided the planetary system's size remains constant relative to the star cluster radial scale).

The results have been applied to three typical cases of clusters, the high-density old globular cluster 47 Tuc, the intermediate density young star forming cluster in the Orion nebula (ONC), and the low-density open cluster of the Hyades. We find that even in the dense environment of 47 Tuc about one quarter of the short-period planets survive even after 10 Gyrs. Our results are interesting for a number of reasons. First of all, the diffusive nature of encounters between planetary systems and stars has been confirmed. With the exception of the few close encounters most intermediate and distant encounters have no preferred sign of change for the orbital parameters of the planet. Therefore planets can be scattered onto a more eccentric orbit, even if they are initially very close to the host star. Subsequent evolution of such planets by tidal effects may lead to their inflation and tidal disruption. This process is expected to be particularly effective in dense globular clusters. Therefore it is not guaranteed that the fraction of surviving systems we have found for the case of 47 Tuc would be observable - it may just be destroyed by secondary processes such as tidal interactions. Here we have just rescaled from our exemplary model simulations to the 
particle number and mass of 47 Tuc; a detailed case study of 47 Tuc would require a model using the half-mass radius and concentration parameter properly tailored so as to reproduce the presently observed values. Also, a more detailed case study of 47 Tuc would require as well to include a stellar mass spectrum, stellar evolution, some binary fraction. This is subject of future work and beyond the scope of this first exploratory study.

In contrast to the less-rich Taurus star forming region, the ONC contains massive stars similar to the hypothetical progenitors which generated the radioactive isotopes prior to the formation of the solar system. This and other, slightly less rich associations are also likely to disperse in $\sim 100 \mathrm{Myr}$ rather than evolve into an open cluster. The main issue here is whether the planet-bearing stars can preserve their companions before joining the field star population. Our numerical results indicate that in the ONC a few times $10^{8}$ years is a critical threshold for the survival of wider planets and planetary systems such as the solar system. Thus, the disruption of some planetary systems is expected, especially near the region where dense protostellar cores are concentrated. These are also the regions where the existence of free floaters have been reported. Our models suggest that a significant fraction of the freefloater population may represent the relics of disrupted planetary systems. Our results also indicate that the velocity dispersion of the freely floating planets must be generally small, as most are retained by the cluster potential, at least for the case of soft planets (Tables 2 and 3 ).

Finally, for open clusters such as the Hyades, there is no problem in preventing all the planetary systems (wide or compact) from destruction before the cluster dissolves on a Gyr time scale. The observed dichotomy in the fraction of stars with planets between the field stars and the Hyades requires additional explanation. The present-day age of the Hyades is $\tau_{\text {Hya }} \simeq 0.8$ Gyr. It is likely that the cluster was somewhat denser in the past which would shorten the planetary-system disruption time scale below that listed in Table 4. A more compact young Hyades would also reduce its $\tau_{\mathrm{ff}}$ below its lifespan. Both eccentricity excitation and modest migration would shake up otherwise stable planetary systems. If systems are driven into nearly resonant configurations, modest eccentricity excitation can render them dynamically unstable. We have not yet taken into account the dynamics of multi-planetary systems in our model, but this subject will be addressed in our future investigations.

Last, but not least, stellar encounters with planetary system can alter the eccentricity of orbits with relatively large semi-major axes efficiently, and also they are the only process which can create significant amounts of inclinations out of the original orbital plane of the planetary system (though we have not considered that issue in this paper). These properties are potentially important for understanding the formation of Kuiper-Belt objects.

We have neglected for the present paper the effect of stellar binaries and multiples, as 
well as that of a stellar mass spectrum. In particular the presence of a large number of binaries would be expected to have a profound effect through the action of binary system - planetary system encounters. The inclusion of binary stars would certainly reduce the magnitude of $\tau_{\mathrm{ff}}$ and the key issue is by what extent. Also a further study of the free floaters in globular and open clusters is interesting. Due to their very small mass they cannot receive large amounts

of energy in the mass segregation - equipartition processes structuring the cluster during its dynamical evolution. Therefore large numbers of free floating planets should be retained in the cluster, a conclusion in conflict at least with present observations. From the point of view of escape, free-floating planets would behave very much like low-mass stars. There is much observational and theoretical evidence that low-mass objects escape preferentially from star clusters, especially in the presence of steady and time-dependent tidal fields. Mass segregation tends to drive such objects to large radii, where they may be efficiently removed by tidal effects. To study the fate of a component of very low-mass planetary objects during the standard evolution of star cluster is another interesting future project.

We conclude that, for an understanding of the diversity of planetary systems, the fact that they originate in a dense star cluster cannot be neglected. Our solar system could have formed in an open cluster or in the outskirts of an object which evolved from an ONC like star cluster. Other processes shaping planetary systems, such as resonances, and interaction with gas, need to be distinguished from the effect of diffusive encounters.

\section{Acknowledgments}

RS and DNCL acknowledge support from a DAAD funded personal project partnership. RS wishes to thank Doug Lin and collegues at the UC Santa Cruz and the Kavli Institute for Astronomy and Astrophysics in Beijing, China for extraordinary kind and friendly support and hospitality, and support of the Global Networks Funds of Heidelberg University is appreciated. The collaboration of MG and RS has been made possible by a cooperation grant of German Science Foundation under 436 POL 113/103. This research was supported in part by NASA (NAGS5-11779, NNG04G-191G, NNG06-GH45G), JPL(1270927), NSF(AST0507424, PHY99-0794) and by the Polish National Committee for Scientific Research under grant 1 P03D 002 27. DCH warmly thanks MG for his hospitality during a visit to Warsaw which greatly facilitated his contribution to this project. Simulations were performed on the GRACE supercomputer (Grants I/80041-043 of the Volkswagen Foundation and 823.219439/30 and /36 of the Ministry of Science, Research and the Arts of Baden-W"urttemberg) and on the IBM Jump supercomputer at NIC Jülich. We thank the referee, John Fregeau, for a careful and constructive review of the paper. 


\section{REFERENCES}

Aarseth S.J. 1999a, Cel. Mech. Dyn. Astron. 73, 127

Aarseth, S.J. 1999b, PASP 111, 1333

Aarseth, S.J. 2003, Gravitational N-Body Simulations, Cambridge Univ. Press, Cambridge

Adams, F. C., Laughlin, G., Icarus 150, 151 (2001)

Armitage, P.J., Livio, M., Lubow, S. H., Pringle, J. E. 2002, MNRAS, 324, 248

Beckwith, S.V.W., Science With The NGST (Next Generation of Space Telescope), edited by Eric P. Smith and Anuradha Koratkar, ASP Conference Series vol. 133, 1998, p. 152

Bonnell, Ian A., Smith, Kester W., Davies, Melvyn B., Horne, K., 2001, MNRAS, 322, 859

Bryden, G., Różyczka, M., Lin, D.N.C., \& Bodenheimer, P. 2000, ApJ 540, 1091

Cameron, A. G. W., Truran, J. W., Icarus 30, 447 (1977)

Correia, A. C. M., Udry, S., Mayor, M., Eggenberger, A., Naef, D., Beuzit, J. -., Perrier, C., Queloz, D., Sivan, J. -., Pepe, F., Santos, N. C., Segransan, D., ArXiv e-prints 711, arXiv:0711.3343 (2007)

Davies, M.B., \& Sigurdsson, S. 1991, MNRAS 324, 612

Duncan, M.J., \& Quinn, T. 1993, ARAA 31, 265

Fischer, D. A., Marcy, G. W., Oppenheimer, B. R., Quirrenbach, A., Frink, S., Monet, D. G., Scargle, J., Butler, R. P., 2001, American Astronomical Society, 198th AAS Meeting; Bulletin of the American Astronomical Society, Vol. 33, p.878

Fregeau, J. M., Chatterjee, S., \& Rasio, F. A., 2006, ApJ, 640, 1086

Funato, Y., Hut, P., McMillan, S.L.W., Makino, J. 1996, AJ 112, 1697

Gao, B., Goodman, J., Cohn, H., Murphy, B., 1991, ApJ, 370, 567

Giersz, M., \& Heggie, D.C., 1994, MNRAS 268, 257

Giersz, M., \& Spurzem, R. 2000, MNRAS 317, 581

Giersz, M., \& Spurzem, R. 2003, MNRAS 343, 781 
Gilliland, R.L., Brown, T. M., Guhathakurta, P., Sarajedini, A., Milone, E. F., Albrow, M. D., Baliber, N. R., Bruntt, H., Burrows, A., Charbonneau, D. et al., 2000, ApJ, 545, L47

Gu, P., Lin, D.N.C., \& Bodenheimer, P.H., 2003, ApJ, 588, 509

Guenther, E. W., Paulson, D. B., Cochran, W. D., Patience, J., Hatzes, A. P., Macintosh, B., Astronomy and Astrophysics 442, 1031 (2005)

Harris W. E., 1996, AJ, 112, 1487

Hatzes, A. P., Cochran, W. D., Endl, M., McArthur, B., Paulson, D. B., Walker, G. A. H., Campbell, B., Yang, S., Astrophysical Journal 599, 1383 (2003)

Han C., 2006, ApJ, 644, 1232

Heggie, D.C., 1975, MNRAS, 173, 729

Heggie, D.C., 2005, astro-ph/0512504

Heggie, D. C., \& Hut, P. 1993, ApJS, 85, 347

Heggie, D. C., \& Mathieu, R. D. 1986, The Use of Supercomputers in Stellar Dynamics, 267, 233

Heggie, D. C., \& Rasio, F. A. 1996, MNRAS, 282, 1064

Hillenbrand, L. A., ArXiv Astrophysics e-prints, arXiv:astro-ph/0511083 (2005)

Hillenbrand, L. A., Hartmann, L. W., Astrophysical Journal 492, 540 (1998)

Hurley J. R., Shara M. M., 2002, ApJ, 565, 1251

Hut, P., Makino, J., \& McMillan, S.L.W. 1995, ApJ 443, L93

Ida, S., \& Lin, D. N. C., 2005, ApJ, 626, 1045

Kokubo, E., Yoshinaga, K., \& Makino, J. 1998, MNRAS, 297, 1067

Laughlin, G, \& Adams, F.C., 1998, ApJ, 508. L171

Lin, D.N.C., Bodenheimer, P., \& Richardson, D. C. 1996, Nature 380, 606

Lin, D. N. C. \& Ida, S., 1997, ApJ, 477, 781

Lucas, P.W., Roche, P.F., Allard, F., Hauschildt, P.H., 2001, MNRAS, 326, 695 
Louis, P.D. \& Spurzem, R., 1991, MNRAS, 251, 408

Marcy, G.W., Butler, R.P., Fischer, D.A., Vogt, S.S., Wright, J.T., Tinney, C.G., Jones, H.R.A., Prog. Theor. Physics Suppl., 2005, 158, 24

Mikkola, S. 1997, CeMDA 67, 145

Mikkola, S., \& Aarseth, S.J. 1998, NewA 3, 309

O’dell, C. R., Wen, Z., Hu, X., Astrophysical Journal 410, 696 (1993)

Perryman, M. A. C., Brown, A. G. A., Lebreton, Y., Gomez, A., Turon, C., de Strobel, G. C., Mermilliod, J. C., Robichon, N., Kovalevsky, J., Crifo, F., Astronomy and Astrophysics 331, 81 (1998)

Roy, A., \& Haddow, M. 2003, Celestial Mechanics and Dynamical Astronomy, 87, 411

Sato, B., Izumiura, H., Toyota, E., Kambe, E., Takeda, Y., Masuda, S., Omiya, M., Murata, D., Itoh, Y., Ando, H., Yoshida, M., Ikoma, M., Kokubo, E., Ida, S., Astrophysical Journal 661, 527 (2007)

Sigurdsson, S., Richer, H.B., Hansen, B.M., Stairs, I.H. \& Thorsett, S.E., 2003, Science, 301, 193

Smith, K.W., \& Bonnell, I.A., 2001, MNRAS, 322, L1

Spurzem, R. 1999, Jl. Comp. Appl. Math. 109, 407

Spurzem, R., \& Giersz, M. 1996, MNRAS, 283, 805

Theuns, T. 1996, MNRAS, 279, 827

Trilling, D. E., Benz, W., Guillot, T., Lunine, J. I., Hubbard, W. B. Burrows, A., 1998, ApJ, 500,428

Weldrake, D. T. F. 2007, astro-ph:0709.4493

Wisdom, J., \& Holman M. 1991, AJ 102, 1520

Zapatero-Osorio, M. R., Bjar, V. J. S., Martn, E. L., Rebolo, R., Barrado Y Navascus, D., Bailer-Jones, C. A. L., Mundt, R., 11th Cambridge Workshop on Cool Stars, Stellar Systems and the Sun, ASP Conference Proceedings, Edited by Ramon J. Garcia Lopez, Rafael Rebolo, and Maria Rosa Zapaterio-Osorio. San Francisco: Astronomical Society of the Pacific, 2000, Vol. 223, p. 70 
Zhou, J.L., Lin, D.N.C., Sun, Y.-S., 2007, ApJ 666, 423

Zucker, D. B., Kniazev, A. Y., Bell, E. F., Martìnez-Delgado, D., Grebel, E. K., Rix, H.-W., Rockosi, C. M., Holtzman, J. A., Walterbos, R. A. M., Annis, J., York, D. G., Ivezic̀ , Z., Brinkmann, J., Brewington, H., Harvanek, M., Hennessy, G., Kleinman, S. J., Krzesinski, J., Long, D., Newman, P. R., Nitta, A., Snedden, S. A., Astrophysical Journal 612, L121 (2004)

\section{A. Formulae of three-body scattering for planetary problems}

We present formulae for the change in binding energy and eccentricity of a binary as a result of scattering by a distant third body. This is a comprehensive collection of main results from earlier papers (Heggie 1975, Heggie \& Rasio 1996, Roy \& Haddow 2003, Heggie 2005), complemented by a few new results, in order to match the requirements for comparison with our numerical simulations.

These formulae assume that the encounter is adiabatic, but take account of the hyperbolic geometry of the relative orbit. In adiabatic encounters we assume that the encounter between the host and field stars occurs on a much longer time scale than the planet's orbital period. Modification of the perturbing potential due to the passage of the field star is essentially adiabatic. Consequently, changes of the eccentricity $\delta e$ can be computed with an orbit-averaging method, in which incremental change of $\delta e$ per orbit can be evaluated for an instantaneous field star position. For $\Delta$, however, the analytical technique is a bit more subtle (see the original papers).

\section{A.1. Change of eccentricity}

\section{A.1.1. Non-circular binaries}

Long ago Heggie (1975), equation (5.66), derived a formula for the change in eccentricity of a binary subject to a parabolic flyby of a third body. Corrected for an overall sign error, it is:

$$
\delta e=-\frac{15 \pi}{4 \sqrt{2}} \frac{m_{3}}{\sqrt{M_{12} M_{123}}} e \sqrt{1-e^{2}}\left(\frac{a}{r_{p}}\right)^{3 / 2}(\hat{\mathbf{a}} \cdot \hat{\mathbf{A}} \hat{\mathbf{b}} \cdot \hat{\mathbf{A}}+\hat{\mathbf{a}} \cdot \hat{\mathbf{B}} \hat{\mathbf{b}} \cdot \hat{\mathbf{B}}),
$$


where $e$ is the eccentricity, $m_{3}$ is the mass of the perturber, $M_{12}=m_{1}+m_{2}$ is the total mass of the binary, $M_{123}=m_{1}+m_{2}+m_{3}$ is the total mass of the system, $a$ is the (initial) semi-major axis of the binary, $r_{p}$ is the distance of closest approach between the perturber and the centre of mass of the binary (on a Keplerian approximation), $\hat{\mathbf{a}}$ is a unit vector along the pericentre of the binary, $\hat{\mathbf{b}}$ is an orthogonal unit vector in the plane of motion of the binary (so that $\hat{\mathbf{a}} \wedge \hat{\mathbf{b}}$ is directed along the angular momentum vector of the binary), $\hat{\mathbf{A}}$ is a unit vector along the pericentre of the third body, and $\hat{\mathbf{B}}$ is an orthogonal unit vector in the plane of motion of the third body (so that $\hat{\mathbf{A}} \wedge \hat{\mathbf{B}}$ is directed along the angular momentum vector of the relative motion of the third body and the binary). Heggie \& Rasio (1996) gave the corresponding formula for a hyperbolic flyby:

$$
\begin{aligned}
\delta e= & -\frac{5 e}{2 e^{\prime^{2}}} \sqrt{\frac{m_{3}^{2} a^{3}\left(1-e^{2}\right)}{M_{12} M_{123} r_{\mathrm{p}}^{3}\left(1+e^{\prime}\right)^{3}}} \times \\
& \times\left\{\hat{\mathbf{a}} \cdot \hat{\mathbf{A}} \hat{\mathbf{b}} \cdot \hat{\mathbf{A}}\left[3 e^{\prime^{2}} \arccos \left(-\frac{1}{e^{\prime}}\right)+\left(4 e^{\prime^{2}}-1\right) \sqrt{e^{\prime 2}-1}\right]\right. \\
& \left.+\hat{\mathbf{a}} \cdot \hat{\mathbf{B}} \hat{\mathbf{b}} \cdot \hat{\mathbf{B}}\left[3 e^{\prime^{2}} \arccos \left(-\frac{1}{e^{\prime}}\right)+\left(2 e^{\prime^{2}}+1\right) \sqrt{e^{\prime^{2}}-1}\right]\right\}
\end{aligned}
$$

where $e^{\prime}$ is the eccentricity of the third body. Expressing the unit vectors in the equation above using orbital elements as in Roy \& Haddow (2003), equation (18) (angles $\omega, \Omega, i$ ) we can write the result as follows:

$$
\begin{aligned}
\delta e= & -\frac{15}{4}\left(\frac{m_{3}^{2}}{M_{12} M_{123}}\right)^{1 / 2}\left(\frac{a}{r_{p}}\right)^{3 / 2} \frac{e \sqrt{1-e^{2}}}{\left(1+e^{\prime}\right)^{3 / 2}} \times \\
& \times\left\{\sin ^{2} i \sin (2 \Omega)\left[\arccos \left(-1 / e^{\prime}\right)+\sqrt{e^{\prime 2}-1}\right]+\right. \\
& \left.+\frac{1}{3}\left[\left(1+\cos ^{2} i\right) \cos (2 \omega) \sin (2 \Omega)+2 \cos i \sin (2 \omega) \cos (2 \Omega)\right] \frac{\left(e^{\prime 2}-1\right)^{3 / 2}}{e^{\prime 2}}\right\}
\end{aligned}
$$

where $\Omega$ is the longitude of the ascending node of the orbit of the third body, measured in the plane of motion of the binary from a particular origin; $i$ is the inclination of the two orbits; and $\omega$ is the longitude of pericentre of the third body, measured from the ascending node. Thus we have arrived at equation (7) of Heggie \& Rasio (1996). In Section 2.2 and Appendix B] we use this expression to estimate cross sections.

\section{A.1.2. Circular binaries}

The above formulae are all that is needed for most purposes, but for the sake of exposition we also give here two formulae, also from Heggie \& Rasio (1996), for the case when 
$e=0$ initially. For hyperbolic encounters the result is:

$$
\begin{aligned}
\delta e= & 3 \sqrt{2 \pi} \frac{m_{3} M_{12}^{1 / 4}}{M_{123}^{5 / 4}}\left(\frac{r_{\mathrm{p}}}{a}\right)^{3 / 4} \frac{\left(e^{\prime}+1\right)^{3 / 4}}{e^{\prime^{2}}} \times \\
& \times \exp \left[-\left(\frac{M_{12}}{M_{123}}\right)^{1 / 2}\left(\frac{r_{\mathrm{p}}}{a}\right)^{3 / 2} \frac{\sqrt{e^{\prime 2}-1}-\arccos \left(1 / e^{\prime}\right)}{\left(e^{\prime}-1\right)^{3 / 2}}\right] \times \\
& \times \cos ^{2} \frac{i}{2}\left[\cos ^{4} \frac{i}{2}+\frac{4}{9} \sin ^{4} \frac{i}{2}+\frac{4}{3} \cos ^{2} \frac{i}{2} \sin ^{2} \frac{i}{2} \cos (4 \omega+2 \Omega)\right]^{1 / 2},
\end{aligned}
$$

This result simplifies a little for parabolic motion of the third body, to

$$
\begin{aligned}
\delta e= & 3 \sqrt{2 \pi} \frac{m_{3} M_{12}^{1 / 4}}{M_{123}^{5 / 4}}\left(\frac{2 r_{\mathrm{p}}}{a}\right)^{3 / 4} \exp \left[-\frac{2}{3}\left(\frac{2 M_{12}}{M_{123}}\right)^{1 / 2}\left(\frac{r_{\mathrm{p}}}{a}\right)^{3 / 2}\right] \times \\
& \times \cos ^{2} \frac{i}{2}\left[\cos ^{4} \frac{i}{2}+\frac{4}{9} \sin ^{4} \frac{i}{2}+\frac{4}{3} \cos ^{2} \frac{i}{2} \sin ^{2} \frac{i}{2} \cos (4 \omega+2 \Omega)\right]^{1 / 2} .
\end{aligned}
$$

\section{A.2. Change of binding energy}

\section{A.2.1. Non-circular binaries}

Roy \& Haddow (2003) give the following expression for the change in energy of the binary in the case of a parabolic flyby:

$$
\begin{aligned}
\delta \varepsilon= & -\frac{G m_{1} m_{2} m_{3}}{M_{12} r_{p}^{3}} \frac{\sqrt{\pi}}{120} K^{5 / 2} \exp (-2 K / 3) \times \\
& \times\left\{60 a^{2} e_{1}\left(\sin \left(n t_{0}\right)(\hat{\mathbf{a}} \cdot \hat{\mathbf{B}})^{2}+2 \cos \left(n t_{0}\right) \hat{\mathbf{a}} \cdot \hat{\mathbf{A}} \hat{\mathbf{a}} \cdot \hat{\mathbf{B}}-\sin \left(n t_{0}\right)(\hat{\mathbf{a}} \cdot \hat{\mathbf{A}})^{2}\right)+\right. \\
& +120 a b e_{4}\left(-\sin \left(n t_{0}\right) \hat{\mathbf{b}} \cdot \hat{\mathbf{B}} \hat{\mathbf{a}} \cdot \hat{\mathbf{A}}-\sin \left(n t_{0}\right) \hat{\mathbf{a}} \cdot \hat{\mathbf{B}} \hat{\mathbf{b}} \cdot \hat{\mathbf{A}}+\right. \\
& \left.+\cos \left(n t_{0}\right) \hat{\mathbf{a}} \cdot \hat{\mathbf{B}} \hat{\mathbf{b}} \cdot \hat{\mathbf{B}}-\cos \left(n t_{0}\right) \hat{\mathbf{a}} \cdot \hat{\mathbf{A}} \hat{\mathbf{b}} \cdot \hat{\mathbf{A}}\right)+ \\
& \left.+60 b^{2} e_{2}\left(\sin \left(n t_{0}\right)(\hat{\mathbf{b}} \cdot \hat{\mathbf{A}})^{2}-2 \cos \left(n t_{0}\right) \hat{\mathbf{b}} \cdot \hat{\mathbf{B}} \hat{\mathbf{b}} \cdot \hat{\mathbf{A}}-\sin \left(n t_{0}\right)(\hat{\mathbf{b}} \cdot \hat{\mathbf{B}})^{2}\right)\right\},
\end{aligned}
$$

where $K=\sqrt{\frac{2 M_{12} r_{p}^{3}}{M_{123} a^{3}}}, b=a \sqrt{1-e^{2}}$ is the semi-minor axis of the binary, $n=\sqrt{\frac{G M_{12}}{a^{3}}}$ is the mean motion of the binary, $t_{0}$ is the time of pericentric passage of the binary (on a time scale in which closest approach of the third body occurs at $t=0$ ), and the coefficients are given by:

$$
\begin{aligned}
& e_{1}=J_{-1}(e)-2 e J_{0}(e)+2 e J_{2}(e)-J_{3}(e) \\
& e_{2}=J_{-1}(e)-J_{3}(e)
\end{aligned}
$$




$$
\begin{aligned}
& e_{3}=e J_{-1}(e)-2 J_{0}(e)+2 J_{2}(e)-e J_{3}(e) \\
& e_{4}=J_{-1}(e)-e J_{0}(e)-e J_{2}(e)+J_{3}(e)
\end{aligned}
$$

here $J_{n}$ is the Bessel function of the first kind of order $n$.

By combining the mathematical procedures in the two papers Heggie \& Rasio (1996) and Roy \& Haddow (2003) it is not hard to show that the corresponding result for a hyperbolic encounter of eccentricity $e^{\prime}$ gives the following expression:

$$
\begin{aligned}
\delta \varepsilon= & -\frac{G m_{1} m_{2} m_{3}}{M_{12} a^{3}} \frac{\sqrt{2 \pi}}{120}\left(\frac{M_{12}}{M_{123}}\right)^{5 / 4}\left(\frac{r_{p}}{a}\right)^{3 / 4} \frac{\left(e^{\prime}+1\right)^{3 / 4}}{e^{\prime 2}} \\
& \exp \left(-\frac{n}{n^{\prime}}\left(\sqrt{e^{\prime 2}-1}-\arccos \frac{1}{e^{\prime}}\right)\right)\left\{6 0 a ^ { 2 } e _ { 1 } \left(\sin \left(n t_{0}\right)(\hat{\mathbf{a}} \cdot \hat{\mathbf{B}})^{2}+\right.\right. \\
& \left.+2 \cos \left(n t_{0}\right) \hat{\mathbf{a}} \cdot \hat{\mathbf{A}} \hat{\mathbf{a}} \cdot \hat{\mathbf{B}}-\sin \left(n t_{0}\right)(\hat{\mathbf{a}} \cdot \hat{\mathbf{A}})^{2}\right)+ \\
& +120 a b e_{4}\left(-\sin \left(n t_{0}\right) \hat{\mathbf{b}} \cdot \hat{\mathbf{B}} \hat{\mathbf{a}} \cdot \hat{\mathbf{A}}-\sin \left(n t_{0}\right) \hat{\mathbf{a}} \cdot \hat{\mathbf{B}} \hat{\mathbf{b}} \cdot \hat{\mathbf{A}}+\right. \\
& +\cos \left(n t_{0}\right) \hat{\mathbf{a}} \cdot \hat{\mathbf{B}} \hat{\mathbf{b}} \cdot \hat{\mathbf{B}}- \\
& \left.-\cos \left(n t_{0}\right) \hat{\mathbf{a}} \cdot \hat{\mathbf{A}} \hat{\mathbf{b}} \cdot \hat{\mathbf{A}}\right)+60 b^{2} e_{2}\left(\sin \left(n t_{0}\right)(\hat{\mathbf{b}} \cdot \hat{\mathbf{A}})^{2}-\right. \\
& \left.\left.-2 \cos \left(n t_{0}\right) \hat{\mathbf{b}} \cdot \hat{\mathbf{B}} \hat{\mathbf{b}} \cdot \hat{\mathbf{A}}-\sin \left(n t_{0}\right)(\hat{\mathbf{b}} \cdot \hat{\mathbf{B}})^{2}\right)\right\}
\end{aligned}
$$

here $n^{\prime}=\sqrt{\frac{G M_{123}}{a^{\prime 3}}}$, where $a^{\prime}$ is the semi-major axis of the hyperbolic motion, and so $r_{p}=a^{\prime}\left(e^{\prime}-1\right)$. Now expressing again the result by using orbital elements we get:

$$
\begin{aligned}
\delta \varepsilon= & -\frac{G m_{1} m_{2} m_{3}}{M_{12} r_{p}^{3}} \frac{\sqrt{\pi}}{8} \frac{\left(e^{\prime}+1\right)^{3 / 4}}{2^{3 / 4} e^{\prime 2}} K^{5 / 2} \exp \left(-\frac{K}{\sqrt{2}}\left(\frac{\sqrt{e^{\prime 2}-1}-\arccos \left(1 / e^{\prime}\right)}{\left(e^{\prime}-1\right)^{3 / 2}}\right)\right) \times \\
& \times\left\{e _ { 1 } a ^ { 2 } \left[\sin \left(2 \omega+n t_{0}\right)(\cos (2 i)-1)-\sin \left(2 \omega+n t_{0}\right) \cos (2 \Omega) \cos (2 i)-\right.\right. \\
& \left.-3 \sin \left(2 \omega+n t_{0}\right) \cos (2 \Omega)-4 \cos \left(2 \omega+n t_{0}\right) \sin (2 \Omega) \cos i\right]+ \\
& +e_{2} b^{2}\left[\sin \left(2 \omega+n t_{0}\right)(1-\cos (2 i))-\sin \left(2 \omega+n t_{0}\right) \cos (2 \Omega) \cos (2 i)-\right. \\
& \left.-3 \sin \left(2 \omega+n t_{0}\right) \cos (2 \Omega)-4 \cos \left(2 \omega+n t_{0}\right) \sin (2 \Omega) \cos i\right]+ \\
& +e_{4} a b\left[-2 \cos \left(2 \omega+n t_{0}\right) \sin (2 \Omega) \cos (2 i)-6 \cos \left(2 \omega+n t_{0}\right) \sin (2 \Omega)-\right. \\
& \left.\left.-8 \sin \left(2 \omega+n t_{0}\right) \cos (2 \Omega) \cos i\right]\right\}
\end{aligned}
$$

where $\Omega$ is measured from â. From here the interested reader should read off the definition of the functions $F, f_{1}$, and $f_{2}$ used in equations (24) and (25).

\section{A.2.2. Circular binaries, parabolic case}

Roy and Haddow (2003) also provided an expression for the energy change of a circular binary in the case of parabolic flyby. This formula, expressed in terms of the orbital elements, 
is as follows:

$$
\begin{aligned}
\delta \varepsilon= & -\frac{G m_{1} m_{2} m_{3}}{M_{12}} \frac{\sqrt{\pi} a^{3}}{8 r_{p}^{4}} K^{7 / 2} \exp \left(-\frac{2}{3} K\right)\left(\mu_{2}-\mu_{1}\right)(1+\cos i) \sin ^{2} i \quad \times \\
& \times\left[\left(\cos ^{3} \omega-3 \sin ^{2} \omega \cos \omega\right) \sin \left(n t_{0}\right)+\left(3 \cos ^{2} \omega \sin \omega-\sin ^{3} \omega\right) \cos \left(n t_{0}\right)\right]
\end{aligned}
$$

where $\mu_{i}=m_{i} / M_{12} \quad(i=1,2)$ are the reduced masses.

\section{B. Cross sections for the change of eccentricity}

\section{B.1. Impulsive tidal encounters}

Here our starting point is an expression for the total change, due to the passing star, in the specific angular momentum $J$ of the planet relative to its sun. Following Heggie (1975), where (in Section 4.2) an analogous calculation of the energy change is given, we obtain

$$
\delta \mathbf{J}=\frac{2 G m}{V} \mathbf{r} \times\left(\frac{\mathbf{p}-\mathbf{r}_{\perp}}{\left|\mathbf{p}-\mathbf{r}_{\perp}\right|^{2}}-\frac{\mathbf{p}}{|\mathbf{p}|^{2}}\right) .
$$

Here $\mathbf{p}$ is a vector from the sun to the closest point of the (rectilinear) path of the passing star, and $\mathbf{r}_{\perp}$ is the component of the position vector of the planet, at this time, which is orthogonal to the path of the passing star. As usual we have specialised to the case of equal stellar mass and negligible planetary mass.

In the tidal regime we expand in powers of $\mathbf{r}_{\perp}$, obtaining to lowest order

$$
\delta \mathbf{J}=\frac{2 G m}{V p^{2}} \mathbf{r} \times\left(-\mathbf{r}_{\perp}+2\left(\mathbf{r}_{\perp} \hat{\mathbf{p}}\right) \hat{\mathbf{p}}\right)
$$

where $\hat{\mathbf{p}}$ is the unit vector parallel to $\mathbf{p}$. Hence

$$
\mathbf{J} . \delta \mathbf{J}=-\frac{2 G m}{V p^{2}} \mathbf{v} . \mathbf{r} \times\left(\mathbf{r} \times\left(-\mathbf{r}_{\perp}+\left(\mathbf{r}_{\perp} \hat{\mathbf{p}}\right) \hat{\mathbf{p}}\right)\right) .
$$

The corresponding result for the change in energy, $\delta \varepsilon$, is similar, except that the factor $\mathbf{r} \times$ in equation (B2) is replaced by $\mathbf{v} \cdot$, where $\mathbf{v}$ is the velocity of the planet (relative to its sun) at the time of closest approach of the passing star. By equation (6) the change in $e^{2}$ can now be written as

$$
D=\frac{4 r_{\perp}}{G m V p^{2}} \mathbf{A}_{\perp} \cdot\left(-\hat{\mathbf{r}_{\perp}}+2 \hat{\mathbf{r}_{\perp}} \hat{\mathbf{p}} \hat{\mathbf{p}}\right)
$$

where $\mathbf{A}=J^{2} \mathbf{v}+2 \varepsilon \mathbf{J} \times \mathbf{r}$. Now $\mathbf{A}_{\perp}, \mathbf{r}_{\perp}$ and $\hat{\mathbf{p}}$ are vectors in the same plane, and so $D=$ $-\frac{4 r_{\perp} A_{\perp}}{G m V p^{2}} \cos (\psi+2 \theta)$, where $\theta, \psi$ are the angles in this plane from $\hat{\mathbf{r}_{\perp}}$ to $\hat{\mathbf{p}}, \mathbf{A}_{\perp}$, respectively. 
Thus the differential cross section, before averaging all the binary parameters, is

$$
\frac{d \sigma}{d D}=\int \delta\left(D+\frac{4 r_{\perp} A_{\perp}}{G m V p^{2}} \cos (\psi+2 \theta)\right) p d p d \theta
$$

Hence

$$
\begin{aligned}
\frac{d \sigma}{d D} & =\int \frac{p d p}{\sqrt{\left(\frac{4 r_{\perp} A_{\perp}}{G m V p^{2}}\right)^{2}-D^{2}}} \\
& =\frac{2 r_{\perp} A_{\perp}}{G m V D^{2}} .
\end{aligned}
$$

Next we note that $r_{\perp}$ scales as $a$, the semi-major axis, and $A$ scales with $(G m)^{3 / 2} a^{1 / 2}$. Hence we can write

$$
\frac{d \sigma}{d D}=\frac{2(G m)^{1 / 2} a^{3 / 2}}{V D^{2}} r_{\perp 1} A_{\perp 1}
$$

the extra subscripts denoting these normalisations.

This is as far as we have been able to take the analytic evaluation of this cross section. Resorting to Monte Carlo integration to average over the normalised binary parameters, we found that

$$
\frac{d \sigma}{d D}=\frac{2(G m)^{1 / 2} a^{3 / 2} C_{1}}{V D^{2}}
$$

where $C_{1}=0.883$ to three significant figures.

\section{B.2. Extremely hyperbolic adiabatic encounters}

We begin with equation (A2), let $m_{1}=m_{3}=m$ and $m_{2}=0$, and take the asymptotic form for $e^{\prime} \rightarrow \infty$. In this limit $r_{p} \simeq p$, the impact parameter, and we evaluate the limiting expression for $e^{\prime}$ using equation (2). The result is

$$
\delta e=-\frac{5 e \sqrt{G m\left(1-e^{2}\right)} a^{3 / 2}}{V p^{2}}(2 \hat{\mathbf{a}} \cdot \hat{\mathbf{A}} \hat{\mathbf{b}} \cdot \hat{\mathbf{A}}+\hat{\mathbf{a}} \hat{\mathbf{B}} \hat{\mathbf{b}} \cdot \hat{\mathbf{B}}),
$$

where $\hat{\mathbf{a}}, \hat{\mathbf{b}}$ are certain orthogonal unit vectors in the plane of motion of the planet, and $\hat{\mathbf{A}}, \hat{\mathbf{B}}$ are similarly defined in the plane of motion of the passing star (Appendix A.1.1). Hence the total cross section for $D$ is, for $D_{0}>0$,

$$
\sigma\left(D>D_{0}\right)=\pi p^{2}=-\frac{10 \pi e^{2} \sqrt{G m\left(1-e^{2}\right)} a^{3 / 2}}{D V}(2 \hat{\mathbf{a}} \cdot \hat{\mathbf{A}} \hat{\mathbf{b}} \cdot \hat{\mathbf{A}}+\hat{\mathbf{a}} \hat{\mathbf{B}} \hat{\mathbf{b}} \cdot \hat{\mathbf{B}})
$$


provided that the last factor is negative.

Now we average over the orientation of the planetary orbit. To do this we think of $\hat{\mathbf{A}}$ and $\hat{\mathbf{B}}$ as providing the first two vectors of a basis, and the expression to be averaged is therefore $X=2 \hat{a}_{1} \hat{b}_{1}+\hat{a}_{2} \hat{b}_{2}$, where this is negative. With the usual spherical polar coordinates we take

$$
\hat{\mathbf{a}}=(\sin \theta \cos \phi, \sin \theta \sin \phi, \cos \theta),
$$

and then $\hat{\mathbf{b}}$, which is an arbitrary unit vector orthogonal to $\hat{\mathbf{a}}$, may be written as

$$
\hat{\mathbf{b}}=(\cos \theta \cos \phi \cos \psi-\sin \phi \sin \psi, \cos \theta \sin \phi \cos \psi+\cos \phi \sin \psi,-\sin \theta \cos \psi) \text {. }
$$

Therefore $X=\alpha \cos \psi+\beta \sin \psi$, where

$$
\begin{aligned}
\alpha & =\sin \theta \cos \theta\left(2 \cos ^{2} \phi+\sin ^{2} \phi\right) \\
\beta & =-\cos \phi \sin \phi \sin \theta
\end{aligned}
$$

Averaging with respect to $\psi$ for $X<0$ gives

$$
\langle X\rangle=-\frac{2}{\pi} \sqrt{\alpha^{2}+\beta^{2}}
$$

It appears that averaging over $\theta$ and $\phi$ has to be done by numerical quadrature, and yields

$$
\langle X\rangle=-\frac{2 C_{2}}{\pi}
$$

where $C_{2}=0.5932$ approximately.

Finally we average over a thermal distribution of $e$, and differentiate with respect to $D$ to obtain the differential cross section

$$
\frac{d \sigma}{d D}=\frac{16 C_{2}}{3} \frac{\sqrt{G m a^{3}}}{V D^{2}}
$$

\section{B.3. Near-parabolic encounters}

We begin with equation (19) in Heggie \& Rasio (1996). Specialising to the case of two equal masses $m$ and one vanishing mass, this becomes

$$
\sigma\left(\delta e>\delta e_{0}\right)=\frac{9 \sqrt{3}}{14}\left(\frac{15 \pi}{2}\right)^{2 / 3}\left[\Gamma\left(\frac{2}{3}\right) \Gamma\left(\frac{5}{6}\right)\right]^{2} \frac{G m a}{V^{2}} e^{2 / 3}\left(1-e^{2}\right)^{1 / 3}\left(\delta e_{0}\right)^{-2 / 3} .
$$


Eliminating $\delta e$ in favour of $D=\delta\left(e^{2}\right) \simeq 2 e \delta e$, and then differentiating with respect to $D$, we arrive at the differential cross section

$$
\frac{d \sigma}{d D}=\frac{3 \sqrt{3}}{7 \pi}(15 \pi)^{2 / 3}\left[\Gamma\left(\frac{2}{3}\right) \Gamma\left(\frac{5}{6}\right)\right]^{2} \frac{G m a}{V^{2}} e^{4 / 3}\left(1-e^{2}\right)^{1 / 3}(D)^{-5 / 3} .
$$

Finally, averaging over a thermal distribution of eccentricities, i.e. $f(e)=2 e$, gives

$$
\frac{d \sigma}{d D}=\frac{2}{21}(15 \pi)^{2 / 3}\left[\Gamma\left(\frac{2}{3}\right) \Gamma\left(\frac{5}{6}\right)\right]^{2} \frac{G m a}{V^{2}}(D)^{-5 / 3} .
$$

\section{Cross Sections for the Change in Energy}

Here we consider only adiabatic encounters, and turn first to the parabolic case. Equation (A6) shows that

$$
\Delta=g \exp \left(-\frac{2 K}{3}\right)
$$

where $K=\left(r_{p} / a\right)^{3 / 2}$ for the present assumptions about the masses, and $g$ is a dimensionless coefficient which depends on $\mathrm{K}$ and on the geometry of the encounter. Remarkably, we do not need to evaluate it in order to obtain the cross section to lowest order. Using equation (A6) in the parabolic limit, we deduce that

$$
\sigma=\frac{\pi p^{2}}{2}=\frac{2 \pi G m a}{V^{2}}\left(-\frac{3}{2} \ln \frac{\Delta}{g}\right)^{2 / 3},
$$

where the factor of $1 / 2$ in the second expression is needed because the geometric factor $g$ has the correct sign in only half of all encounters. Differentiating with respect to $\Delta$, we can ignore the derivative of $g ; g$ depends on powers of $r_{p}$ or $p$ and hence on powers of $\ln \Delta$, as we can see from equation ( $(\mathrm{C2})$, and so it varies much more slowly than $\Delta$. Similarly we can neglect $\ln |g|$ by comparison with $|\Delta|$ for sufficiently weak encounters. Therefore

$$
\frac{d \sigma}{d \Delta}=\frac{2 \pi G m a}{V^{2}} \frac{1}{|\ln | \Delta||^{1 / 3}|\Delta|}
$$

The extremely hyperbolic case follows a similar line of argument. As stated in equation (27) we have

$$
\Delta=g \exp \left(-\frac{2 K}{3 \sqrt{e^{\prime}}}\right) .
$$

Now we use equations (2) and (4) to express everything in terms of $p$, and find that

$$
\sigma=\frac{\pi p^{2}}{2}=\frac{\pi V^{2} a^{3}}{2 G m}(\ln (\Delta / g))^{2} .
$$


Differentiation with respect to $\Delta$ with the same approximations leads to the result

$$
\frac{d \sigma}{d \Delta}=\frac{\pi a^{3} V^{2}}{G m} \frac{|\ln | \Delta||}{|\Delta|} .
$$

